\title{
A Mountaineering Strategy to Excited States: Highly-Accurate Energies and Benchmarks for Exotic Molecules and Radicals
}

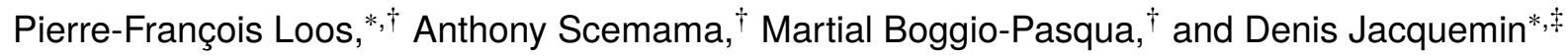 \\ $\dagger$ Laboratoire de Chimie et Physique Quantiques, Université de Toulouse, CNRS, UPS, France \\ $\ddagger$ Université de Nantes, CNRS, CEISAM UMR 6230, F-44000 Nantes, France \\ E-mail: loos@irsamc.ups-tlse.fr; Denis.Jacquemin@univ-nantes.fr
}

\begin{abstract}
Aiming at completing the sets of FCI-quality transition energies that we recently developed (J. Chem. Theory Comput. 14 (2018) 4360-4379, ibid. 15 (2019) 1939-1956, and ibid. 16 (2020) 1711-1741), we provide, in the present contribution, ultra-accurate vertical excitation energies for a series of "exotic" closed-shell molecules containing $\mathrm{F}, \mathrm{Cl}, \mathrm{P}$, and $\mathrm{Si}$ atoms and small radicals, such as $\mathrm{CON}$ and its variants, that were not considered to date in such investigations. This represents a total of 81 high-quality transitions obtained with a series of diffuse-containing basis sets of various sizes. For the exotic compounds, these transitions are used to perform benchmarks with a vast array of lower-level models, i.e., CIS(D), EOM-MP2, (SOS/SCS)-CC2, STEOM-CCSD, CCSD, CCSDR(3), CCSDT-3, (SOS-)ADC(2), and ADC(3). Additional comparisons are made with literature data. For the open-shell compounds, we have compared the performances of both the unrestricted and restricted open-shell CCSD and CC3 formalisms.
\end{abstract}

\section{INTRODUCTION}

The increase of computational ressources coupled to the emergence of more advanced algorithms has led to a resurgence of the selected configuration interaction (SCI) approaches ${ }^{1-3}$ as an effective strategy to rapidly reach the full CI (FCI) limit at a fraction of the cost of a genuine FCI calculation thanks to a sparse exploration of the FCI space. ${ }^{4-19}$ This revival is especially beneficial for the calculation of transition energies between electronic states, ${ }^{6,15-23}$ as the accurate determination of these energies remains one of the great challenges faced by theoretical chemists.

Recently, we have developed two sets of theoretical best estimates (TBEs) of FCI quality for the vertical transition energies of small closed-shell compounds. ${ }^{20,21}$ (See Ref. 24 for a recent review.) In our first work, ${ }^{20}$ we reported TBEs for more than 100 electronic transitions of single-excitation character in organic compounds containing from one to three non-hydrogen atoms, namely $\mathrm{C}, \mathrm{N}, \mathrm{O}$, and $\mathrm{S}$. These TBEs have been obtained thanks to an efficient implementation of the CIPSI (Configuration Interaction using a Perturbative Selection made Iteratively) SCI algorithm, ${ }^{19}$ which selects the most important determinants in the FCI space using a secondorder perturbative criterion. ${ }^{8}$ Their quality was further confirmed by equation-of-motion coupled-cluster (CC) calculations performed up to high excitation degrees. It turned out that $\mathrm{CC}$ including contributions up to the quadruples (CCSDTQ $)^{25}$ yields transition energies almost systematically equal to FCI, with a mean absolue error (MAE) as small as $0.01 \mathrm{eV}$, whereas the three tested $\mathrm{CC}$ approaches including perturbative triples, namely, CC $3,{ }^{26,27}$ CCSDT- $3,{ }^{28,29}$ and
$\mathrm{CCSDT}^{30}$ are also very effective with MAEs of $0.03 \mathrm{eV} .{ }^{20}$ This means that these four CC models are (on average) chemically accurate (error smaller than $1 \mathrm{kcal}^{\mathrm{mol}}{ }^{-1}$ or $0.043 \mathrm{eV}$ ) for these single excitation transitions.

Our second set encompasses 20 transitions characterized by a large and/or dominant double excitation nature. ${ }^{21}$ These types of electronic excitations are known to be much more challenging for single-reference methods. For this set, we relied again on SCI methods to determine TBEs and we evaluated the performances of various multi-reference approaches, such as the second-order complete active space perturbation theory (CASPT2), ${ }^{31,32}$ and the second-order $n$ electron valence state perturbation theory (NEVPT2) ${ }^{33-35}$ methods. Interestingly, for excitations with a large but not dominant double excitation character, such as the first ${ }^{1} A_{g}$ excited state of trans-butadiene, it turns out that the accuracy obtained with CC 3 and NEVPT2 are rather similar with MAEs of ca. $0.12 \mathrm{eV}^{21}$ In contrast, for genuine double excitations (i.e, excitations with an insignificant amount of single excitation character) in which one photon effectively promotes two electrons, the $\mathrm{CC} 3$ error becomes extremely large (of the order of $1 \mathrm{eV}$ ) and multi-reference approaches have clearly the edge (for example, the MAE of NEVPT2 is 0.07 $\mathrm{eV}){ }^{21}$

To the very best of our knowledge, these two sets taken together constitute the largest ensemble of chemically-accurate vertical transition energies published to date with roughly 130 transition energies of FCI quality. Despite their decent sizes and the consideration of both valence and Rydberg excited states, these sets have obvious limitations. Let us point 
out four of these biases: (i) only small compounds are included; (ii) some important classes of transitions, such as charge-transfer (CT) excitations, are absent; (iii) compounds including only $\mathrm{C}, \mathrm{N}, \mathrm{O}, \mathrm{S}$, and $\mathrm{H}$ atoms have been considered; (iv) these sets include only singlet-singlet and singlet-triplet excitations in closed-shell molecules.

Very recently, we have made extensive efforts in order to solve the first limitation. ${ }^{23}$ However, performing SCI or high-level CC calculations rapidly becomes extremely tedious when one increases the system size as one hits the exponential wall inherently linked to these methods. At this stage, we believe that circumventing the second limitation is beyond reach as clear intramolecular CT transitions only occur in (very) large molecules for which CCSDTQ or SCI calculations remain clearly out of reach with current technologies. We note, however, that intermolecular CT energies were recently obtained at the CCSDT level by Kozma and coworkers. ${ }^{36}$ Therefore, the aim of the present contribution is to get rid of the two latter biases. To this end, we consider here (i) a series of closed-shell compounds including (at least) one of the following atoms: $\mathrm{F}, \mathrm{Cl}, \mathrm{Si}$, or $\mathrm{P}$; (ii) a series of radicals characterized by open-shell electronic configurations and an unpaired electron. For the sake of simplicity, we denote the first additional set as "exotic" because it includes a series of chemical species that are rather unusual for organic chemistry, e.g, $\mathrm{H}-\mathrm{P}=\mathrm{S}$ and $\mathrm{H}_{2} \mathrm{C}=\mathrm{Si}$. Similar compounds were included in a benchmark set by the Ortiz group. ${ }^{37}$ They were, however, using experimental data as reference, which often precludes straightforward comparisons with theoretical vertical transition energies. ${ }^{38,39}$ On the other hand, the second set, simply labeled as "radical", encompasses doubletdoublet transitions in radicals. We believe that the additional FCI-quality estimates that we provide in the present study for both types of compounds nicely complete our previous works and will be valuable for the electronic structure community.

\section{COMPUTATIONAL METHODS}

Our computational protocol closely follows the one of Ref. 20. Consequently, we only report key elements below. We refer the reader to our previous work for further information about the methodology and the technical details. ${ }^{20}$ In the following, we report several statistical indicators: the mean signed error (MSE), mean absolute error (MAE), root-mean square error (RMSE), and standard deviation of the errors (SDE).

\subsection{Geometries and basis sets}

For the exotic set, we use CC3/aug-cc-pVTZ ground-state geometries obtained without frozen-core (FC) approximation (i.e, correlating all electrons) to be consistent with our previously-published geometries. ${ }^{20,40-42}$ These optimizations have been performed using DALTON $2017^{43}$ and CFOUR 2.1, ${ }^{44}$ applying default parameters. For the open-shell derivatives, the geometries are optimized at the UCCSD(T)/aug-cc-pVTZ level using the GAUSSIAN16 program $^{45}$ and applying the TIGHT convergence threshold. The Cartesian coordinates of each compound are available in the Supporting Information (SI).

Throughout this paper, we use either the diffuse-containing Pople 6-31+G(d) basis set, or the Dunning aug-cc-pVXZ (X
$=\mathrm{D}, \mathrm{T}, \mathrm{Q}$, and 5) correlation-consistent family of atomic bases.

\section{$2.2 \mathrm{CC}$ reference calculations}

The CC calculations are performed with several codes. For closed-shell molecules, CC $3^{26,27}$ calculations are achieved with DALTON ${ }^{43}$ and CFOUR; ${ }^{44}$ CCSDT calculations are performed with CFOUR $^{44}$ and MRCC 2017; ${ }^{46,47}$ the latter code being also used for CCSDTQ and CCSDTQP. Note that all our excited-state $\mathrm{CC}$ calculations are performed within the equation-of-motion (EOM) or linear-response (LR) formalism that yield equivalent excited-state energies. The reported oscillator strengths have been computed in the LR-CC3 formalism only. For open-shell molecules, the CCSDT, CCSDTQ, and CCSDTQP calculations performed with $\mathrm{MRCC}^{46,47}$ do consider an unrestricted Hartree-Fock (UHF) wave function as reference. All excited-state calculations are performed, except when explicitly mentioned, in the FC approximation using large cores for the third-row atoms. All electrons are correlated for the Be atom, for which we systematically applied the basis set as included in MRCC. ${ }^{48}$ (We have noted differences in the definition of the Dunning bases for this particular atom depending on the software that one considers.)

\subsection{Selected Configuration Interaction}

All the SCI calculations are performed within the FC approximation using QUANTUM PACKAGE ${ }^{19}$ where the CIPSI algorithm $^{3}$ is implemented. Details regarding this specific CIPSI implementation can be found in Refs. 19 and 49. We use a state-averaged formalism which means that the ground and excited states are described with the same number and same set of determinants, but different CI coefficients. The SCI energy is defined as the sum of the variational energy (computed via diagonalization of the CI matrix in the reference space) and a second-order perturbative correction which estimates the contribution of the determinants not included in the CI space. ${ }^{8}$ By extrapolating this second-order correction to zero, one can efficiently estimate the FCI limit for the total energies, and hence, compute the corresponding transition energies. We estimate the extrapolation error by the difference between the transition energies obtained with the largest SCI wave function and the FCI extrapolated value. These errors are systematically reported in the Tables below. Although this cannot be viewed as a true error bar, it provides a rough idea of the quality of the FCI extrapolation and estimate.

\subsection{Other wave function calculations}

Our benchmark effort consists in evaluating the accuracy of vertical transition energies obtained at lower levels of theory. These calculations are performed with a variety of codes. For the exotic set, we rely on: GAUSSIAN ${ }^{45}$ and TURBOMOLE $7.3^{50}$ for CIS(D); ${ }^{51,52}$ Q-CHEM 5.2 53 for EOM-MP2 [CCSD(2)] ${ }^{54}$ and ADC(3); ${ }^{55-57}$ Q-CHEM $^{53}$ and TURBOMOLE ${ }^{50}$ for ADC(2); ${ }^{57,58}$ DALTON $^{43}$ and TURBOMOLE $^{50}$ for CC2; ${ }^{59,60}$ DALTON $^{43}$ and GAUSSIAN for CCSD; ${ }^{61}$ DALTON $^{43}$ for CCSDR(3); ${ }^{62} \mathrm{CFOUR}^{44}$ for CCSDT-3; ${ }^{28,29}$ and ORCA ${ }^{63}$ for similarity-transformed EOM-CCSD (STEOM-CCSD). ${ }^{64,65}$ In addition, we evaluate the spin-opposite scaling (SOS) variants of ADC(2), SOS- 
$\mathrm{ADC}(2)$, as implemented in both Q-CHEM,${ }^{66}$ and TURBOMOLE. ${ }^{67}$ Note that these two codes have distinct SOS implementations, as explained in Ref. 66. We also test the SOS and spin-component scaled (SCS) versions of CC2, as implemented in TURBOMOLE. ${ }^{50,67}$ Discussion of various spin-scaling schemes can be found elsewhere. ${ }^{68}$ When available, we take advantage of the resolution-of-the-identity (RI) approximation in TURBOMOLE and Q-CHEM. For the STEOM-CCSD calculations, it was checked that the active character percentage was, at least, $98 \%$. When comparisons between various codes/implementations were possible, we could not detect variations in the transition energies larger than $0.01 \mathrm{eV}$. For the radical set molecules, we applied both the $\mathrm{U}$ (unrestricted) and RO (restricted open-shell) versions of CCSD and CC 3 as implemented in the PSI 4 code, ${ }^{69}$ to perform our benchmarks.

\section{RESULTS AND DISCUSSION \\ 3.1 Exotic set}

3.1.1 Reference values and comparison to literature

Our main results are listed in Table 1 for the exotic set that encompasses 30 electronic transitions (19 singlets and 11 triplets) in 14 molecules containing between two and five non-hydrogen atoms. Before briefly discussing the compounds individually, let us review some general trends. First, as one could expect for rather low-lying excitations, the augcc-pVTZ basis set is sufficient large to provide excitation energies close to the complete basis set (CBS) limit ${ }^{22}$ and the FC approximation is rather unimportant. Indeed, CC3 calculations performed with quadruple- and quintuple- $\zeta$ basis sets, with and without correlating the core electrons for the former basis, yield negligible changes as compared to the aug-cc-pVTZ results. As more quantitatively illustrated by the results gathered in Table $\mathrm{S} 1$ of the SI, the maximal variation between CC3/aug-cc-pVTZ and CC3/aug-cc-pVQZ excitation energies is $0.03 \mathrm{eV}\left({ }^{1} \Delta\right.$ state of $\left.\mathrm{HCP}\right)$, and the MAE between the two basis sets is as small as $0.01 \mathrm{eV}$. The same observation applies to the FC approximation with a mean absolute variation of $0.02 \mathrm{eV}$ between the CC3(full)/aug-ccpCVQZ and CC3(FC)/aug-cc-pVQZ excitation energies. We therefore do not discuss further the quadruple- and quintuple$\zeta$ results in the following, although basis set corrected TBEs can be found in Table 1. Secondly, it can be seen, from the $\mathrm{CC} 3 \% T_{1}$ values (which provides a measure of the amount single excitation character of the considered transition) listed in Table 1, that all the transitions considered here are largely dominated by single excitations, the smallest $\% T_{1}$ being $88 \%$ (the second transition of silylidene). Such character is favorable to ensure a rapid convergence of the CC series. This is clearly exemplified by the convergence behavior of the 6-31+G(d) excitation energies for which the CCSDTQ and the CCSDTQP transition energies are equal for the 11 cases for which the latter level of theory was achievable. Likewise, one notices that the CCSDTQ estimate systematically falls within $0.01 \mathrm{eV}$ of the FCI value that comes with a very small error bar for most transitions. It is also reassuring to see that, for a given basis set, we could not detect variations larger than $0.04 \mathrm{eV}$ between CCSDTQ results and their CC3 and CCSDT counterparts, the changes being typically of ca. $0.01-0.02 \mathrm{eV}$. All these facts indicate that one can trust the FCI estimates, and hence the TBEs listed in Table 1 (for the larger difluorodiazirine molecule, see discussion below).

In the spirit of the famous Thiel paper, ${ }^{70}$ let us now briefly discuss each compound and compare the results to available data. We do not intend here to provide an exhaustive review of previous calculations, which would lead to a gigantic list of references for the triatomic systems, but rather to pinpoint the "best" published excitation energies to date.

Carbonylfluoride. For this compound encompassing four heavy atoms, the convergence of the SCI approach is rather slow and one notices a $0.03 \mathrm{eV}$ drop of the transition energies between CC3 and CCSDT. We therefore used FCI estimates determined with small bases, corrected for basis set effects to generate our TBEs. For the lowest singlet, that is heavily blueshifted as compared to the parent formaldehyde, the most advanced previous theoretical studies reported vertical transition energies of $7.31 \mathrm{eV}[\operatorname{CCSDR}(3)],{ }^{71}$ and 7.31 $\mathrm{eV}[\mathrm{MRCI}+\mathrm{Q}] .{ }^{72}$ The measured EEL value is ca. $7.3 \mathrm{eV},{ }^{72}$ whereas the UV spectrum shows a peak at $7.34 \mathrm{eV} .{ }^{73}$ All these values are obviously compatible with the current result. Note that the interpretation of the measured $0-0$ values for $\mathrm{F}_{2} \mathrm{C}=\mathrm{O}^{74}$ is challenging, as discussed elsewhere. ${ }^{38}$ For the triplet, the previous TBE is likely a $7.07 \mathrm{eV}$ MRCI+Q result, ${ }^{72}$ also very close to our present value, whereas there also exists estimates of the triplet adiabatic energies. ${ }^{75}$

$\mathrm{CCl}_{2}, \mathrm{CClF}$, and $\mathrm{CF}_{2}$. Dichlorocarbene is large enough to make the convergence of the SCI calculations difficult with the triple- $\zeta$ basis, and our TBEs are based on the FCI/augcc-pVDZ values corrected for basis set effects determined at the CC level. While both CC3 and CCSDT almost perfectly reproduce the FCI results for the singlet and triplet $B_{1}$ states, more significant differences are noted for the higher-lying $A_{2}$ states that seem slightly too low with CCSDT. This is also confirmed by the CCSDTQ results obtained with the Pople basis set. Previous calculations are available at CCSD, ${ }^{76}$ and MRCI ${ }^{77,78}$ levels. The most recent MRCI+Q values, obtained with a large atomic basis set are 2.61, 4.49, 1.25 and $4.43 \mathrm{eV}$ for the ${ }^{1} B_{1},{ }^{1} A_{2},{ }^{3} B_{1}$, and ${ }^{3} A_{2}$ transitions, respectively. These values are reasonably close to the present TBEs. For $\mathrm{CClF}$, the most accurate literature value is probably the MRCI+Q/triple- $\zeta$ estimate of $3.59 \mathrm{eV},{ }^{79}$ within 0.03 $\mathrm{eV}$ of our current TBE. For this compound, we are also aware of three previous experimental investigations focussing on its vibronic spectra. ${ }^{80-82} \mathrm{For}_{2} \mathrm{CF}_{2}$, the SCI calculations converge rapidly even with the aug-cc-pVTZ basis and yield TBEs of 5.09 and $2.77 \mathrm{eV}$ for the lowest singlet and triplet transitions. There has been countless experimental and theoretical investigations for this stable carbene, but the most accurate previous estimates of the vertical transition energies are likely the 5.12 and $2.83 \mathrm{eV}$ values, obtained at the MRCI+Q/aug-cc-pVTZ level of theort. ${ }^{83}$

Difluorodiazirine. This cyclopropene analogue is the largest derivative considered herein. There is a remarkable agreement between CC3 and CCSDT values, and the \% $T_{1}$ value is very large for each transition, so that we consider the $\mathrm{CC}$ values to obtain our TBEs. For the ${ }^{1} B_{1}$ and ${ }^{3} B_{1}$ transitions, $\mathrm{FCI} / 6-31+\mathrm{G}(\mathrm{d})$ calculations deliver transition energies of $3.81 \pm 0.01$ and $3.09 \pm 0.01 \mathrm{eV}$, perfectly consistent 
Table 1: Excitation energies (in eV) of the exotic set obtained within the FC approximation. For each transition, we also report, on the left hand side, the LR-CC3/aug-cc-pVTZ oscillator strength, the CC3 single excitation character $\left(\% T_{1}\right)$, and the TBE/aug-cc-pVTZ excitation energy. Except otherwise stated, the latter has been obtained directly at $\mathrm{FCl} /$ aug-cc-pVTZ level. We also provide the TBE/CBS estimate obtained by correcting the TBE/aug-cc-pVTZ value by the difference between CC3/aug-cc-pV5Z and CC3/aug-cc-pVTZ. On the right hand side, one finds the transition energies computed at various levels of theory. T, TQ, and TQP stand for CCSDT, CCSDTQ, and CCSDTQP, respectively.

\begin{tabular}{|c|c|c|c|c|c|c|c|c|c|c|c|c|c|c|c|c|c|}
\hline \multirow{4}{*}{ Carbonylfluoride } & \multirow{4}{*}{$\begin{array}{l}{ }^{1} A_{2} \\
{ }^{3} A_{2}\end{array}$} & \multicolumn{3}{|c|}{ aug-cc-pVTZ } & \multirow{2}{*}{$\begin{array}{l}\text { CBS } \\
\text { TBE }\end{array}$} & \multicolumn{4}{|c|}{$6-31+G(d)$} & \multicolumn{4}{|c|}{ aug-cc-pVDZ } & \multicolumn{4}{|c|}{ aug-cc-pVTZ } \\
\hline & & $f[\mathrm{CC} 3]$ & $\% T_{1}$ & TBE & & $\mathrm{CC} 3$ & $\mathrm{~T}$ & TQ & TQP & $\mathrm{CC} 3$ & $\mathrm{~T}$ & TQ & FCI & $\mathrm{CC} 3$ & $\mathrm{~T}$ & TQ & FCI \\
\hline & & & 91.1 & $7.31^{a}$ & 7.31 & 7.33 & 7.30 & & & 7.34 & 7.31 & & $7.30 \pm 0.04$ & 7.31 & 7.28 & & $7.32 \pm 0.05$ \\
\hline & & & 97.8 & $7.06^{b}$ & 7.07 & 7.03 & 7.00 & & & 7.05 & 7.02 & & $7.08 \pm 0.01$ & 7.03 & 7.00 & & $7.04 \pm 0.10$ \\
\hline \multirow[t]{4}{*}{$\mathrm{CCl}_{2}$} & ${ }^{1} B_{1}$ & 0.002 & 93.7 & $2.59^{b}$ & 2.57 & 2.71 & 2.70 & 2.70 & & 2.69 & 2.69 & & $2.68 \pm 0.02$ & 2.61 & 2.60 & & \\
\hline & ${ }^{1} \mathrm{~A}_{2}$ & & 88.3 & $4.40^{b}$ & 4.41 & 4.46 & 4.44 & 4.47 & & 4.40 & 4.39 & & $4.46 \pm 0.01$ & 4.35 & 4.33 & & \\
\hline & ${ }^{3} B_{1}$ & & 98.6 & $1.22^{b}$ & 1.23 & 1.10 & 1.09 & 1.11 & & 1.20 & 1.19 & & $1.22 \pm 0.03$ & 1.20 & 1.19 & & $1.22 \pm 0.05$ \\
\hline & ${ }^{3} A_{2}$ & & 96.1 & $4.31^{b}$ & 4.32 & 4.41 & 4.38 & 4.42 & & 4.34 & 4.31 & & $4.36 \pm 0.01$ & 4.28 & 4.26 & & \\
\hline $\mathrm{CClF}$ & ${ }^{1} A^{\prime \prime}$ & 0.007 & 93.9 & $3.55^{b}$ & 3.54 & 3.66 & 3.66 & 3.66 & & 3.63 & 3.62 & & $3.62 \pm 0.01$ & 3.56 & 3.55 & & $3.63 \pm 0.06$ \\
\hline \multirow[t]{2}{*}{$\mathrm{CF}_{2}$} & ${ }^{1} B_{1}$ & 0.034 & 94.7 & 5.09 & 5.07 & 5.18 & 5.18 & 5.18 & & 5.12 & 5.11 & 5.11 & $5.12 \pm 0.00$ & 5.07 & 5.06 & & $5.09 \pm 0.01$ \\
\hline & ${ }^{3} B_{1}$ & & 99.1 & 2.77 & 2.78 & 2.71 & 2.70 & 2.71 & & 2.71 & 2.70 & 2.71 & $2.71 \pm 0.01$ & 2.76 & 2.75 & & $2.77 \pm 0.01$ \\
\hline \multirow[t]{6}{*}{ Difluorodiazirine } & ${ }^{1} B_{1}$ & 0.002 & 93.1 & $3.74^{c}$ & $3.73^{d}$ & 3.83 & 3.83 & & & 3.80 & 3.80 & & & 3.74 & 3.74 & & \\
\hline & ${ }^{1} A_{2}$ & & 91.4 & $7.00^{c}$ & $6.98^{d}$ & 7.13 & 7.11 & & & 7.11 & 7.08 & & & 7.02 & 7.00 & & \\
\hline & ${ }^{1} B_{2}$ & 0.026 & 93.3 & $8.52^{c}$ & $8.54^{d}$ & 8.51 & 8.52 & & & 8.45 & 8.46 & & & 8.50 & 8.52 & & \\
\hline & ${ }^{3} B_{1}$ & & 98.2 & $3.03^{e}$ & $3.03^{d}$ & 3.09 & 3.09 & & & 3.06 & 3.06 & & & 3.03 & & & \\
\hline & ${ }^{3} B_{2}$ & & 98.9 & $5.44^{e}$ & $5.46^{d}$ & 5.48 & 5.48 & & & 5.47 & 5.46 & & & 5.45 & & & \\
\hline & ${ }^{3} B_{1}$ & & 98.4 & $5.80^{e}$ & $5.81^{d}$ & 5.86 & 5.85 & & & 5.83 & 5.82 & & & 5.81 & & & \\
\hline \multirow[t]{2}{*}{ Formylfluoride } & ${ }^{1} A^{\prime \prime}$ & 0.001 & 91.2 & $5.96^{b}$ & 5.97 & 6.09 & 6.06 & 6.07 & & 6.03 & 6.00 & & $6.00 \pm 0.03$ & 5.99 & 5.96 & & \\
\hline & ${ }^{3} A^{\prime \prime}$ & & 97.9 & $5.73^{b}$ & 5.75 & 5.72 & 5.70 & 5.71 & & 5.65 & 5.62 & & $5.65 \pm 0.01$ & 5.62 & 5.60 & & \\
\hline $\mathrm{HCCl}$ & ${ }^{1} A^{\prime \prime}$ & 0.003 & 94.5 & 1.98 & 1.97 & 2.05 & 2.04 & 2.05 & 2.05 & 2.02 & 2.02 & 2.02 & $2.04 \pm 0.01$ & 1.97 & 1.97 & & $1.98 \pm 0.00$ \\
\hline $\mathrm{HCF}$ & ${ }^{1} A^{\prime \prime}$ & 0.006 & 95.4 & 2.49 & 2.49 & 2.58 & 2.57 & 2.58 & 2.58 & 2.53 & 2.53 & 2.53 & $2.54 \pm 0.00$ & 2.49 & 2.49 & & $2.49 \pm 0.02$ \\
\hline \multirow[t]{4}{*}{$\mathrm{HCP}$} & ${ }^{1} \Sigma^{-}$ & & 94.9 & 4.84 & 4.81 & 5.19 & 5.19 & 5.18 & 5.18 & 5.06 & 5.05 & 5.04 & $5.04 \pm 0.00$ & 4.85 & 4.85 & 4.84 & $4.84 \pm 0.00$ \\
\hline & ${ }^{1} \Delta$ & & 94.0 & 5.15 & 5.10 & 5.48 & 5.48 & 5.48 & 5.48 & 5.33 & 5.33 & 5.32 & $5.32 \pm 0.00$ & 5.15 & 5.15 & & $5.15 \pm 0.00$ \\
\hline & ${ }^{3} \Sigma^{+}$ & & 98.9 & 3.47 & 3.49 & 3.44 & 3.45 & 3.46 & 3.46 & 3.47 & 3.47 & 3.49 & $3.49 \pm 0.00$ & 3.45 & 3.45 & 3.46 & $3.47 \pm 0.00$ \\
\hline & ${ }^{3} \Delta$ & & 98.8 & 4.22 & 4.20 & 4.40 & 4.39 & 4.39 & 4.39 & 4.35 & 4.34 & 4.34 & $4.34 \pm 0.00$ & 4.22 & 4.21 & 4.21 & $4.22 \pm 0.00$ \\
\hline HPO & ${ }^{1} A^{\prime \prime}$ & 0.003 & 90.9 & 2.47 & 2.49 & 2.49 & 2.47 & 2.48 & 2.48 & 2.47 & 2.45 & 2.46 & $2.46 \pm 0.00$ & 2.46 & 2.46 & & $2.47 \pm 0.00$ \\
\hline HPS & ${ }^{1} A^{\prime \prime}$ & 0.001 & 90.3 & 1.59 & 1.61 & 1.57 & 1.55 & 1.56 & 1.56 & 1.60 & 1.59 & 1.59 & $1.60 \pm 0.00$ & 1.59 & 1.58 & & $1.59 \pm 0.00$ \\
\hline $\mathrm{HSiF}$ & ${ }^{1} A^{\prime \prime}$ & 0.024 & 93.1 & 3.05 & 3.05 & 3.09 & 3.08 & 3.08 & 3.08 & 3.08 & 3.07 & 3.07 & $3.06 \pm 0.00$ & 3.07 & 3.06 & & $3.05 \pm 0.00$ \\
\hline \multirow[t]{2}{*}{$\mathrm{SiCl}_{2}$} & ${ }^{1} B_{1}$ & 0.031 & 92.1 & $3.91^{b}$ & 3.93 & 3.94 & 3.94 & 3.94 & & 3.93 & 3.92 & & $3.95 \pm 0.02$ & 3.90 & 3.88 & & $3.88 \pm 0.03$ \\
\hline & ${ }^{3} B_{1}$ & & 98.7 & $2.48^{f}$ & 2.50 & 2.39 & 2.39 & 2.40 & & 2.45 & 2.44 & & $2.47 \pm 0.05$ & 2.48 & 2.47 & & $2.49 \pm 0.04$ \\
\hline \multirow[t]{2}{*}{ Silylidene } & ${ }^{1} A_{2}$ & & 92.3 & 2.11 & 2.12 & 2.14 & 2.11 & 2.10 & 2.10 & 2.18 & 2.15 & 2.14 & $2.14 \pm 0.00$ & 2.15 & 2.13 & 2.12 & $2.11 \pm 0.01$ \\
\hline & ${ }^{1} B_{2}$ & 0.033 & 88.0 & 3.78 & 3.80 & 3.88 & 3.87 & 3.88 & 3.88 & 3.81 & 3.80 & 3.80 & $3.79 \pm 0.01$ & 3.78 & 3.78 & 3.78 & $3.78 \pm 0.01$ \\
\hline
\end{tabular}

${ }^{a} \mathrm{FCI} / 6-31+\mathrm{G}(\mathrm{d})$ value of $7.33 \pm 0.02 \mathrm{eV}$ corrected by the difference between CCSDT/aug-cc-pVTZ and CCSDT/6-31+G(d); ${ }^{b} \mathrm{FCI} /$ aug-cc-pVDZ value corrected by the difference between CCSDT/aug-cc-pVTZ and CCSDT/aug-cc-pVDZ; ${ }^{c}$ CCSDT/aug-cc-pVTZ value; ${ }^{d}$ Corrected with the quadruple- $\zeta$ basis rather than the quintuple- $\zeta$ basis; ${ }^{e}$ CCSDT/aug-cc-pVDZ value corrected by the difference between CC3/aug-cc-pVTZ and CC3/aug-cc-pVDZ;

${ }^{f}$ CCSDTQ/6-31+G(d) value corrected by the difference between CCSDT/aug-cc-pVTZ and CCSDT/6-31+G(d). 
with the present $\mathrm{CC}$ values. Our TBEs are likely the most accurate to date for vertical transitions. At the GVVPT2/ccpVTZ level, the transition energies reported in Ref. 84 are $2.25 \mathrm{eV}\left({ }^{3} B_{1}\right), 2.95 \mathrm{eV}\left({ }^{1} B_{1}\right), 4.86 \mathrm{eV}\left({ }^{3} B_{2}\right), 5.21 \mathrm{eV}\left({ }^{3} A_{2}\right)$, $6.63 \mathrm{eV}\left({ }^{1} A_{2}\right)$, and $8.23 \mathrm{eV}\left({ }^{1} B_{2}\right)$, which follows exactly the same state ordering as the present CCSDT values. More recently, QCISD/aug-cc-pVTZ estimates of 2.81 and $3.99 \mathrm{eV}$ for the lowest triplet and singlet vertical transitions have been reported, which are respectively slightly smaller and larger than the present data. There are also quite a few studies of the 0-0 energies of various states for this derivative, both experimentally ${ }^{85-87}$ and theoretically. ${ }^{84,88,89}$

Formylfluoride. For this formal intermediate between carbonylfluoride and formaldehyde, we note that the CCSDTQ/6-31+G(d) values are bracketed by their CC3 and CCSDT counterparts. The previous best estimates are likely the very recent MRCI-F12 results of Pradhan and Brown who reported vertical transition energies of $6.03 \mathrm{eV}$ and $5.68 \mathrm{eV}$ for the ${ }^{1} A^{\prime \prime}$ and ${ }^{3} A^{\prime \prime}$ states, respectively. These energies obtained on the CCSD(T)-F12 ground-state geometries are only ca. $0.05 \mathrm{eV}$ larger than the present TBEs. Most other previous studies focussed on $0-0$ energies of the lowest singlet state, ${ }^{38,75,89-95}$ and it is noteworthy that $\mathrm{CC} 3$ reproduces the experimental 0-0 energies with high accuracy. ${ }^{38,89}$ Our TBE for the singlet state $(5.96 \mathrm{eV})$ is much larger than the measured $0-0$ peak $(4.64 \mathrm{eV})^{93}$ which is expected for a molecule undergoing an important geometrical relaxation after excitation. ${ }^{92}$

$\mathrm{HCCl}, \mathrm{HCF}$, and HSiF. For these three compounds, the SCI calculations deliver values very close to the $\mathrm{CC}$ estimates. For $\mathrm{HCCl}$, a MRCI+Q/quintuple- $\zeta$ vertical transition energy, corrected for ground-state ZPVE effects, of $1.68 \mathrm{eV}$ was recently reported. ${ }^{96}$ Given that the ZPVE energy at the MP2/aug-cc-pVTZ level is $0.31 \mathrm{eV}$, our TBE is basically equivalent to this recent result. For $\mathrm{HSiF}$, the most accurate previous estimate of the excitation energy is likely the CC3/aug-cc-pVTZ $3.07 \mathrm{eV}$ value, ${ }^{97}$ which is extremely close to our TBE. For the records, Ehara and coworkers also investigated the $0-0$ energies and excited-state geometries of these three systems at the SAC-CI level, ${ }^{98}$ and experimental $0-0$ energies of $1.52 \mathrm{eV}(\mathrm{HCCl}),{ }^{99} 2.14 \mathrm{eV}(\mathrm{HCF}),{ }^{100,101}$ and $2.88 \mathrm{eV}$ (HSiF), ${ }^{102}$ have been measured.

HCP. Phosphaethyne is a linear compound for which the $\mathrm{CC}$ series and the SCI values do converge rapidly and give equivalent results. Consequently, one can trust the TBEs listed in Table 1. We nevertheless note that there is a significant basis set effect for the ${ }^{1} \Delta$ excited state that is downshifted by $0.05 \mathrm{eV}$ from aug-cc-pVTZ to aug-cc-pV5Z (see Table S1 in the SI). The two most refined previous theoretical works we are aware of have been performed at the MRCI/double- $\zeta^{103}$ and CC3/cc-pVQZ ${ }^{104}$ levels of theory and respectively focussed on reproducing the experimental vibronic couplings and understanding the $\mathrm{HCP} \longrightarrow \mathrm{HPC}$ isomerization process. However, somehow surprisingly, we could not find recent estimates of the vertical transition energies for phosphaethyne, the previously published data being apparently of CASSCF quality. ${ }^{105}$ There are, of course, experimental characterizations of the $0-0$ energies for several excited states of this compound. ${ }^{106}$
HPO and HPS. The lowest excited state of HPO has been studied several times in the last twenty years, ${ }^{89,98,107-109}$ whereas its sulfur analogue has only been considered more recently. ${ }^{89,110-112}$ In both cases, refined MRCI calculations of the vibronic spectra have been performed ${ }^{98,109-112}$ but few reported vertical transition energies. We are aware of a quite old CASPT2 estimate of $2.25 \mathrm{eV}$ for HPO, ${ }^{107}$ and a recent MRCI vertical transition energy of $1.69 \mathrm{eV}$ (obtained with a very large basis set) for HPS. ${ }^{112}$

$\mathrm{SiCl}_{2}$. In this heavier analogue of dichlorocarbene, there are no strong methodological effects but the SCI convergence is shaky, especially for the triplet and we used a basis set extrapolated CCSDTQ value as TBE for this state. Advanced calculations of the adiabatic energies ${ }^{113}$ as well as experimental $0-0$ energies ${ }^{114,115}$ can be found in the literature, the latter being 3.72 and $2.35 \mathrm{eV}$ for the lowest singlet and triplet states, respectively. These values are sightly larger than our vertical estimates. For the vertical singlet excitation, there is also a recent $4.06 \mathrm{eV}$ CCSD//CAM-B3LYP estimate, ${ }^{116}$ which slightly overshoots ours, consistent with the expected error sign of CCSD. ${ }^{20,70,117}$

Silylidene. One notes an excellent agreement between CCSDT, CCSDTQ, and FCI for this derivative. Our TBEs of $2.11 \mathrm{eV}$ and $3.78 \mathrm{eV}$ are again exceeding the experimental 0-0 energies of $1.88 \mathrm{eV}^{118}$ and $3.63 \mathrm{eV},{ }^{119}$ as it should. The previous theoretical studies we are aware of have been performed with CISD(+Q), ${ }^{118,120}$ and CC $3{ }^{38,89}$ methods and mainly discussed the 0-0 energies, for which an excellent agreement with experiment was obtained by both approaches.

\subsubsection{Benchmarks}

Benchmarks using the TBEs obtained in the previous Section can be naturally done. As we consider closed-shell compounds, there is a large number of methods that one can evaluate. Here, we have chosen 15 popular wave function methods for excited states (see Computational Details and Table S2 in the SI for the raw data). The statistical result can be found in Figure 1 and Table 2.

Most of the conclusions that can be extracted from these benchmarks are consistent with recent analyses made in the field, ${ }^{20,23,89,117,121-124}$ and we will therefore only briefly comment on the most significant outcomes. First, one notes that CC3, which is an expensive approach, is superbly accurate and consistent with a trifling MSE and a tiny SDE, whereas both CCSDT-3 and CCSDR(3), for which only singlet excited states can be evaluated with the current implementations, are also extremely satisfying with average errors well below the chemical accuracy threshold. This is unsurprisingly inline with the trends obtained for more "standard" organic compounds: CC methods including (at least partially) contributions from the triples are trustworthy for the description of single excitations. ${ }^{20,89,117,125-128}$ Going down in the CC hierarchy, we find that CCSD slightly overestimates the transition energies, but nevertheless provides very consistent estimates (SDE of $0.08 \mathrm{eV}$ ), whereas CC2 is clearly less satisfying in terms of consistency (SDE of $0.14 \mathrm{eV}$ ). Comparing with previous benchmarks, ${ }^{20,41,65,70,117,121,122,127,129,130}$ we can foresee that the CCSD overestimation will likely grow in larger compounds, whereas the $\mathrm{CC} 2$ accuracy should remain less affected by the system size. The SOS and SCS variants of 

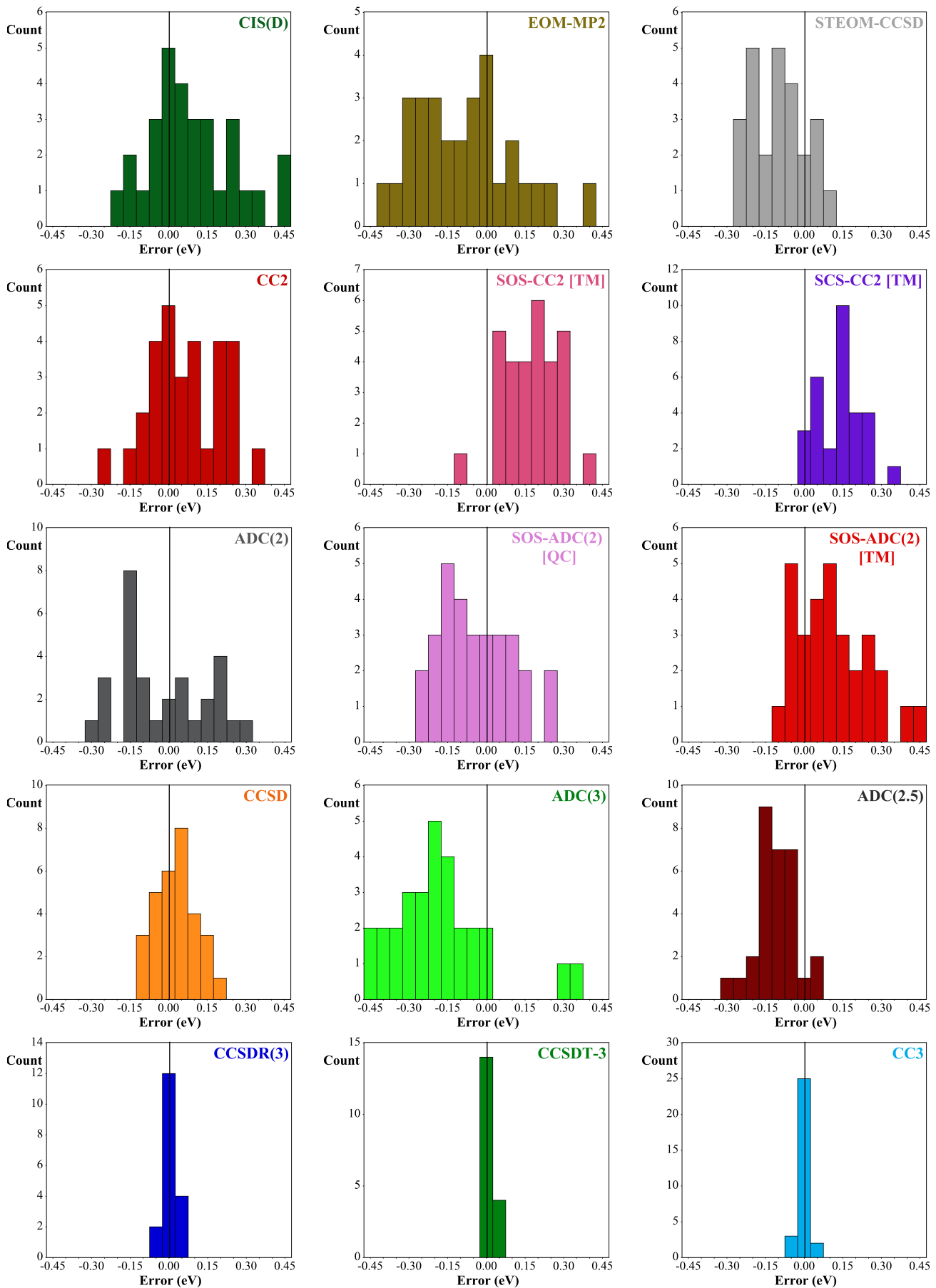

Figure 1: Histograms of the error distribution (in eV) obtained with 15 theoretical methods, choosing the TBE/aug-cc-pVTZ of Table 1 as references. TM and QC stand for the TURBOMOLE and Q-CHEM definitions of the scaling factors, respectively. Note the difference of scaling in the vertical axes. 
Table 2: Statistical values obtained by comparing the results of various methods to the TBE/aug-cc-pVTZ values listed in Table 1. We report the mean signed error (MSE), mean absolute error (MAE), root-mean square error (RMSE), and standard deviation of the errors (SDE). All quantities are given in $\mathrm{eV}$ and have been obtained with the aug-cc-pVTZ basis set. TM and QC stand for the TURBOMOLE and Q-CHEM definitions of the scaling factors, respectively. $A D C(2.5)$ is the simple average of the $A D C(2)$ and $A D C(3)$ transition energies, as defined in Ref. 121. "Count" refers to the number of transitions computed for each method.

\begin{tabular}{lccccc}
\hline Method & Count & MSE & MAE & RMSE & SDE \\
\hline CIS(D) & 30 & 0.09 & 0.14 & 0.19 & 0.16 \\
EOM-MP2 & 30 & -0.06 & 0.17 & 0.22 & 0.21 \\
STEOM-CCSD & 25 & -0.10 & 0.12 & 0.14 & 0.10 \\
CC2 & 30 & 0.07 & 0.12 & 0.15 & 0.14 \\
SOS-CC2 [TM] & 30 & 0.17 & 0.18 & 0.20 & 0.10 \\
SCS-CC2 [TM] & 30 & 0.14 & 0.14 & 0.16 & 0.09 \\
ADC(2) & 30 & -0.02 & 0.15 & 0.16 & 0.17 \\
SOS-ADC(2) [TM] & 30 & 0.11 & 0.13 & 0.17 & 0.14 \\
SOS-ADC(2) [QC] & 30 & -0.04 & 0.12 & 0.14 & 0.14 \\
CCSD & 30 & 0.03 & 0.07 & 0.08 & 0.08 \\
ADC(3) & 30 & -0.19 & 0.24 & 0.27 & 0.19 \\
ADC(2.5) & 30 & -0.11 & 0.11 & 0.13 & 0.07 \\
CCSDR(3) & 19 & 0.01 & 0.02 & 0.02 & 0.02 \\
CCSDT-3 & 19 & 0.01 & 0.02 & 0.02 & 0.02 \\
CC3 & 30 & 0.00 & 0.01 & 0.02 & 0.02 \\
\hline
\end{tabular}

CC2 deliver larger MAE, with a clear overestimation (see Figure 1), but a smaller error dispersion than the standard CC2 method. The accuracy deterioration and the improved consistency of the spin-scaled CC2 versions (w.r.t. standard CC2) is known, ${ }^{68,124,131}$ though some works reported that SOS-CC2 and SCS-CC2 can also improve the accuracy. ${ }^{132}$ STEOMCCSD delivers results of roughly $\mathrm{CC} 2$ quality for the present set, whereas patterns more alike the ones of CCSD have been previously obtained. ${ }^{20,23,65}$ In the present case, both CIS(D) and EOM-MP2 [also denoted CCSD(2)], which are the two computationally lightest approaches, are also the ones yielding the largest dispersions alongside quite significant MAEs. For EOM-MP2, similar outcomes were observed for valence excited states by Tajti and Szalay, ${ }^{123}$ whereas the relatively poor performance of CIS(D) is well documented. ${ }^{20,23,68,131}$ In the $\mathrm{ADC}$ series, we note that $\mathrm{ADC}(2)$ yields results only slightly less accurate than $\mathrm{CC} 2$ for a smaller computational cost, which is consistent with the conclusions of Dreuw's group, ${ }^{56}$ whereas the SOS variant developed by the same group ${ }^{66}$ has a slight edge over its TURBOMOLE variant. ADC (3) provides rather poor excitation energies, a trend we recently evidenced in other molecular sets. ${ }^{20,121,128}$ Finally, the very recently introduced $\mathrm{ADC}(2.5)$ scheme, which corresponds to the simple average of the $\mathrm{ADC}(2)$ and $\mathrm{ADC}(3)$ excitation energies, ${ }^{121}$ provides significantly more consistent estimates than both $\mathrm{ADC}(2)$ or $\mathrm{ADC}(3)$, with a SDE of 0.07 $\mathrm{eV}$ only compared to ca. $0.18 \mathrm{eV}$ for the "parent" methods. $\mathrm{ADC}(2.5)$ can then be seen as a cost effective approach to improve upon $\mathrm{ADC}(3)$, at least for small compounds.

\subsection{Radical set}

\subsubsection{Reference values and comparison to literature}

Let us now turn to radicals. As nicely summarized by Crawford fifteen years ago, ${ }^{133}$ electronic transitions in open-shell systems are more challenging, not only due to the more lim- ited number of methods and codes available for treating them (as compared to closed-shell molecules), but also because: (i) strong spin contamination can take place with "low"-level methods; (ii) large contributions from doubly-excited configurations are quite common; and (iii) basis set effects can be very large, meaning that reaching the CBS limit can be laborious. At the CCSD level for instance, significant differences between $\mathrm{U}$ and RO transition energies can sometimes be observed. ${ }^{133}$ This is why our results, listed in Table 3, use as computationally-lightest approach (U)CCSDT, so that the wave function is robust enough in order to mitigate the two former issues for most of the considered transitions. As can be seen in the 6-31+G(d) and aug-cc-pVDZ columns of Table 3, one generally finds an excellent agreement between the various CC estimates and their FCI counterparts, UCCSDT being already extremely accurate except in specific cases (such as the ${ }^{2} \Sigma^{+}$excited state of $\mathrm{CO}^{+}$). This overall consistency indicates yet again that one can trust the present TBEs. We underline that, except for diatomics, UCCSDT calculations performed with diffuse basis sets on open-shell molecules are quite rare in the literature (see below), and the same obviously holds for higher-order CC. As for the exotic set, we do not intend here to provide an exhaustive list of previous works, but rather to pinpoint a few interesting comparisons with earlier accurate estimates.

Allyl. For the lowest valence $\left(B_{1}\right)$ and Rydberg $\left(A_{1}\right)$ transitions of the allyl radical, the previous TBEs are likely the ROCC3 3.44 and $4.94 \mathrm{eV}$ vertical transition energies obtained by the Crawford group with the aug-cc-pVTZ basis further augmented with molecule-centered functions (mcf). ${ }^{134}$ For the lowest state, a very similar value of $3.43 \mathrm{eV}$ was obtained at the ROCC3 level without mcf. ${ }^{89}$ The present work is the first to report CCSDT and CCSDTQ results. They clearly show that these previous ROCC 3 estimates are very accurate. In addition, our TBEs of 3.39 and $4.99 \mathrm{eV}$ are reasonably consistent with earlier CASPT2 (3.32 and 5.11 $\mathrm{eV})^{135}$ and MRCI (3.32 and $\left.4.68 \mathrm{eV}\right)^{136}$ data. The experimental 0-0 energies have been reported to be $3.07 \mathrm{eV},{ }^{137}$ and $4.97 \mathrm{eV}^{136,138}$ for the ${ }^{2} B_{1}$ and ${ }^{2} A_{1}$ states, respectively. The fact that the experimental $T_{0}$ value is very close to the computed vertical transition energy of the second state is rather surprising, but remains unchanged with the present work.

$B e F$. In this compound, CCSDT delivers transition energies in very good agreement with FCI (and higher CC levels), but one notices a non-negligible basis set effect for the second transition of Rydberg character. This transition becomes significantly mixed in very large basis sets, making a clear attribution difficult. For this derivative (and other diatomics), experimental vertical transition energies can be calculated by analyzing the experimental spectroscopic constants. ${ }^{139}$ Our TBE/aug-cc-pVTZ values of 4.14 and $6.21 \mathrm{eV}$ are obviously close to these measured values of 4.14 and $6.16 \mathrm{eV}$. ${ }^{139}$ For the lowest state, a previous MRCI value of $4.23 \mathrm{eV}$ can be found in the literature. ${ }^{140}$ There is also a recent evaluation of the adiabatic energies for numerous excited states at the $\mathrm{MRCI}+\mathrm{Q}$ level. ${ }^{141}$

$\mathrm{BeH}$. The convergences with respect to both the CC excitation order and the basis set size is extremely fast for this five-electron system. A previous study reports FCI values for 
Table 3: Excitation energies (in eV) of the radical set obtained within the FC approximation. For each state, we report, on the left hand side, the TBE/aug-cc-pVTZ excitation energy obtained directly at the FCI level (except otherwise stated). The TBE/CBS excitation energy is obtained with the largest affordable basis set (see footnotes). On the right hand side, one finds the transition energies computed at various levels of theory. T, TQ, and TQP stand for UCCSDT, UCCSDTQ, and UCCSDTQP, respectively.

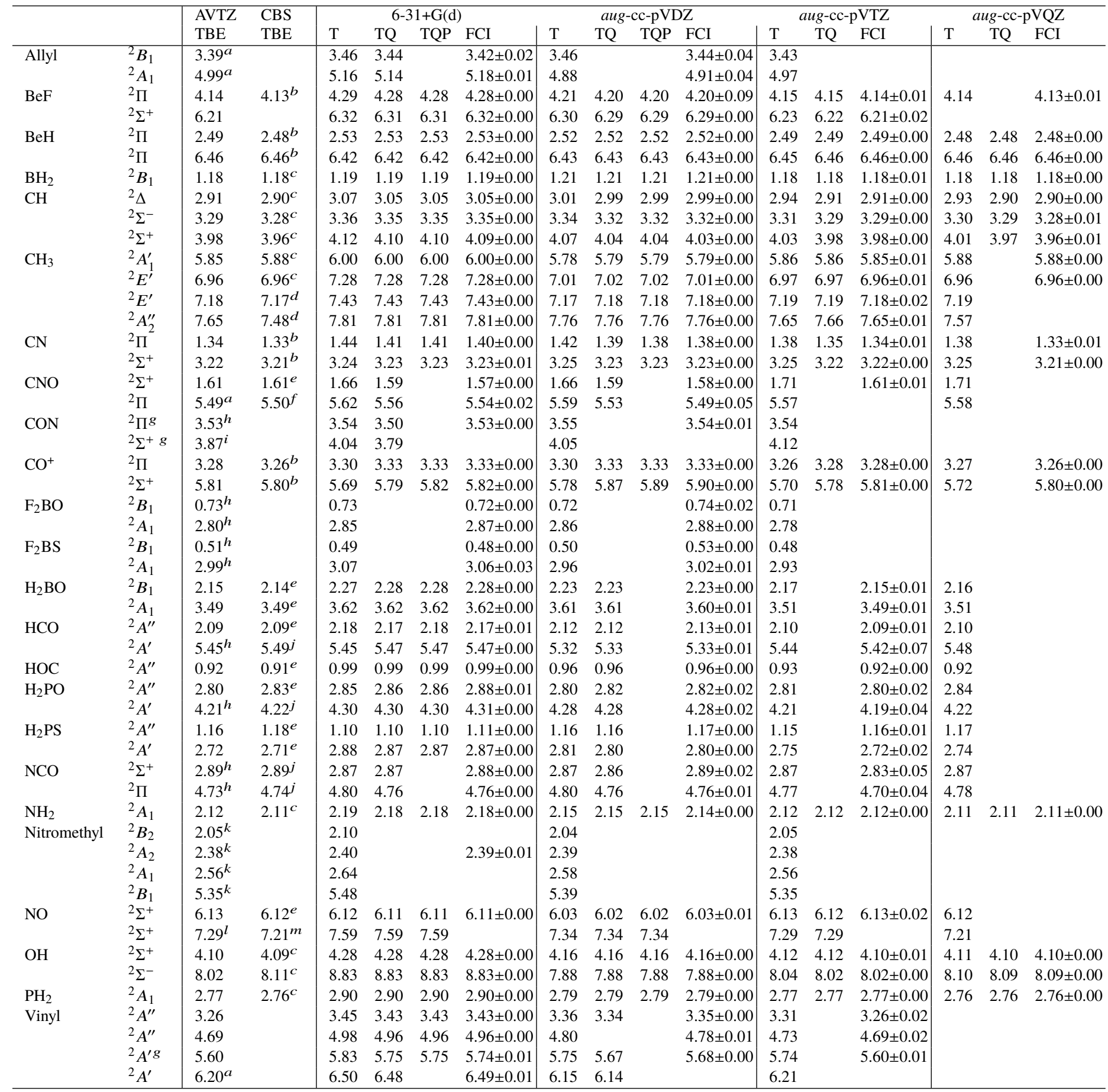

${ }^{a} \mathrm{FCI} / 6-31+\mathrm{G}(\mathrm{d})$ value corrected by the difference between CCSDT/aug-cc-pVTZ and CCSDT/6-31+G(d); ${ }^{b} \mathrm{FCI} /$ aug-cc-pVQZ value; ${ }^{c}$ FCI/aug-cc-pVQZ value corrected by the difference between CCSDT/aug-cc-pV5Z and CCSDT/aug-cc-pVQZ; ${ }^{d}$ FCI/aug-cc-pVTZ value corrected by the difference between CCSDT/aug-cc-pV5Z and CCSDT/aug-cc-pVTZ; ${ }^{e} \mathrm{FCI} /$ aug-cc-pVTZ value corrected by the difference between CCSDT/aug-cc-pVQZ and

CCSDT/aug-cc-pVTZ; ${ }^{f}$ FCI/6-31+G(d) value corrected by the difference between CCSDT/aug-cc-pVQZ and CCSDT/6-31+G(d); ${ }^{g}$ For these challenging states, ROCC rather than UCC is used. ${ }^{h} \mathrm{FCI} /$ aug-cc-pVDZ value corrected by the difference between CCSDT/aug-cc-pVTZ and CCSDT/aug-cc-pVDZ; ${ }^{i} \mathrm{CCSDTQ} / 6-31+\mathrm{G}(\mathrm{d})$ value corrected by the difference between CCSDT/aug-cc-pVTZ and CCSDT/6-31+G(d); ${ }^{j} \mathrm{FCI} /$ aug-cc-pVDZ value corrected by the difference between CCSDT/aug-cc-pVQZ and CCSDT/aug-cc-pVDZ; ${ }^{k}$ CCSDT/aug-cc-pVTZ value; ${ }^{l}$ CCSDTQ/aug-cc-pVTZ value;

${ }^{m} \mathrm{CCSDTQ} /$ aug-cc-pVTZ value corrected by the difference between CCSDT/aug-cc-pVQZ and CCSDT/aug-cc-pVTZ. 
many excited states ${ }^{142}$ and, in particular, excitation energies of 2.53 and $6.30 \mathrm{eV}$ for the two ${ }^{2} \Pi$ states considered herein. The experimental vertical transition energies are 2.48 and $6.32 \mathrm{eV}{ }^{139}$ Our larger value associated with the second transition is likely a consequence of the $\operatorname{UCCSD}(\mathrm{T})$ geometry, which delivers a slightly shorter bond length (1.321 vs 1.327 A experimentally).

$\mathrm{BH}_{2}, \mathrm{NH}_{2}$, and $\mathrm{PH}_{2}$. In these three related compounds, convergence with respect to the $\mathrm{CC}$ excitation order and basis set size is also very fast, so that accurate estimates can be easily produced for the lowest-lying transition: With the augcc-pVTZ basis set, near-CBS excitation energies of 1.18 , 2.12 and $2.77 \mathrm{eV}$ for the boron, nitrogen, and phosphorus derivative are respectively obtained. For $\mathrm{BH}_{2}$, a previous MRCI estimate of $1.10 \mathrm{eV}$ is available in the literature. ${ }^{143}$ We note that, for $\mathrm{BH}_{2}$, the geometry relaxation of the bent ground state structure would lead to a linear geometry in its lowest excited state, ${ }^{144}$ a phenomenon that was extensively studied both experimentally and theoretically (see Ref. 144 and references therein). For $\mathrm{NH}_{2}$, a vertical estimate of 2.18 $\mathrm{eV}$ was reported by Szalay and Gauss using a CCSD approach including "pseudo" triple excitations, ${ }^{145}$ and high-order CC calculations have been latter performed by Kallay and Gauss to investigate the structures and energetics of the ground and excited states. ${ }^{146,147}$ For $\mathrm{PH}_{2}$, the most detailed ab initio studies that are available in the literature focus exclusively on the $0-0$ energies and rovibronic spectra, ${ }^{148-150}$ except for a recent report listing a ROCC3 vertical transition energy of $2.75 \mathrm{eV},{ }^{89}$ obviously close to present TBE.

$\mathrm{CH}$. For the three considered transitions, the CCSDT values are slightly too large, whereas the basis set effects are rather usual, with nearly converged results for the aug-ccpVTZ basis set. Although we consider a theoretical geometry, our basis set corrected TBEs of 2.90, 3.28, and 3.96 $\mathrm{eV}$ for the ${ }^{2} \Delta,{ }^{2} \Sigma^{-}$, and ${ }^{2} \Sigma^{+}$states are all extremely close to the vertical experimental values of $2.88,3.26$ and 3.94 eV. ${ }^{139,151}$ There are many previous works on the $\mathrm{CH}$ radical and it is interesting to mention that the ROCCSD values are $3.21,4.25$, and $5.22 \mathrm{eV}$ for the same three states, ${ }^{145}$ whereas the corresponding ROCC3 results are 3.16, 3.58, and 4.47 $\mathrm{eV} ;{ }^{133}$ the $\operatorname{ROCC}(2,3)$ excitation energies are $2.97,3.33$, and $4.06 \mathrm{eV}{ }^{151}$ This clearly illustrates the challenge of reaching accurate values for the second and third transitions with "loworder" methods. For CH, high-order CC calculations of the adiabatic energies and other properties are also available in the literature. ${ }^{152,153}$

$\mathrm{CH}_{3}$. For the methyl radical, the convergence of the $\mathrm{CC}$ excitation energies and the near-perfect agreement between $\mathrm{CC}$ and FCI is worth noting. Nonetheless, large basis set effects are present for these transition energies, especially for the high-lying ${ }^{2} A_{2}^{\prime \prime}$ state for which the aug-cc-pVTZ excitation energy is still far from being converged basis set wise. Our TBEs, including corrections up to quintuple- $\zeta$ are: $5.88,6.96,7.17$, and $7.48 \mathrm{eV}$ for the four lowest transitions. These values can be compared to the previous MRCI estimates $^{154,155}$ of $5.86(5.91), 6.95(7.03), 7.13$ (-) and 7.37 (7.66) $\mathrm{eV}$ reported in Ref. 154 ( 155$)$. The experimental $T_{0}$ value is $5.73 \mathrm{eV}$ for the ${ }^{2} A_{1}^{\prime}$ state, ${ }^{106,156}$ whereas the experimental $T_{e}$ value is $7.43 \mathrm{eV}$ for the ${ }^{2} A_{2}^{\prime \prime}$ state, ${ }^{157,158}$ both slightly below our FCI vertical estimates.

$C N$. Both methodological and basis set effects are firmly under control for the cyano radical, so that our FCI/aug-ccpVTZ results of 1.34 and $3.22 \mathrm{eV}$ for the lowest excited states are likely very accurate for the considered geometry. These values are indeed close to the experimental energies of 1.32 and $3.22 \mathrm{eV} .^{139}$ One can find careful MRCI studies, ${ }^{159,160}$ as well as an extensive benchmark ${ }^{161}$ for the adiabatic energies of the $\mathrm{CN}$ radical.

$C N O, C O N$, and NCO. Inspired by a previous investigation, ${ }^{159}$ we have evaluated the two lowest doublet transitions in these three linear isomers. For CNO - the second most stable isomer - one notes non-negligible drops of the transition energies going from CCSDT to CCSDTQ, the latter theory providing data in perfect match with the FCI results. Our TBEs of $1.61 \mathrm{eV}\left({ }^{2} \Sigma^{+}\right)$and $5.50 \mathrm{eV}\left({ }^{2} \Pi\right)$, do compare very favorably with the corresponding $\mathrm{MRCI}+\mathrm{Q}$ results of 1.66 and $5.50 \mathrm{eV}$, respectively. ${ }^{159}$ For the former transition, there is also a ROCC3 vertical transition energy of 1.71 $\mathrm{eV}^{89}$ and a detailed rovibronic investigation ${ }^{162}$ available in the literature. The data are much scarcer for $\mathrm{CON}$, and the only previous work we are aware of reports potential energy surfaces without listing explicitly the transition energies. ${ }^{159}$ For CON, we have performed multi-reference calculations to identify the lowest states (see Table S4 in the SI). The NEVPT2 calculations locate the ${ }^{2} \Pi$ and ${ }^{2} \Sigma^{+}$transitions at 3.52 and $3.81 \mathrm{eV}$, respectively, similar values being obtained with both CASPT2 and MRCI. As can be seen in Table 3 the FCI-based estimate of $3.53 \mathrm{eV}$ for the former transition is extremely consistent. For the latter transition, the difference between CCSDT and CCSDTQ energies is as large as $-0.25 \mathrm{eV}$, suggesting that further corrections would be required. Nevertheless, our CC-derived TBE of $3.87 \mathrm{eV}$ is rather consistent with the NEVPT2 and MRCI values. For $\mathrm{NCO}$, the most stable of the three isomers, the basis set effects are trifling, but CCSDTQ is again mandatory in order to obtain a very accurate transition energy for the ${ }^{2} \Pi$ state. This compound was studied previously at the MRCI+Q level, a method which delivers respective vertical transition energies of 2.89 and $4.68 \mathrm{eV}$ for the ${ }^{2} \Sigma^{+}$and ${ }^{2} \Pi$ states, ${ }^{159}$ whereas the ROCC3/aug-cc-pVTZ transition energy of the lowest excited state is $2.83 \mathrm{eV}{ }^{89}$ The measured experimental $0-0$ energies are $2.82,{ }^{163}$ and $3.94 \mathrm{eV} .{ }^{164}$ All these data are quite consistent with our new values of 2.89 and $4.74 \mathrm{eV}$.

$\mathrm{CO}^{+}$. Our FCI/aug-cc-pVQZ values for the ${ }^{2} \Pi$ and ${ }^{2} \Sigma^{+}$ transitions, 3.26 and $5.80 \mathrm{eV}$, are clearly matching the experimental values of 3.26 and $5.81 \mathrm{eV} .{ }^{139}$ While basis set effects are rather standard for this radical cation, it is noteworthy that the CC expansion converges slowly for the Rydberg ${ }^{2} \Sigma^{+}$ transition: one needs CCSDTQP to be within $0.01 \mathrm{eV}$ of the FCI result! Nonetheless, previous ROCC3 (3.29 and 5.73 $\mathrm{eV})^{133}$ and $\operatorname{ROCC}(2,3)$ data $(3.35$ and $5.81 \mathrm{eV}),{ }^{151}$ also fall within $\pm 0.10 \mathrm{eV}$ of the present TBEs.

$\mathrm{F}_{2} \mathrm{BO}$ and $\mathrm{F}_{2} \mathrm{BS}$. These two radicals present a very lowlying $\pi-n$ transition, that is described very similarly by all basis sets used in Table 3. For these transitions our TBEs are $0.73\left(\mathrm{~F}_{2} \mathrm{BO}\right)$ and $0.51\left(\mathrm{~F}_{2} \mathrm{BS}\right) \mathrm{eV}$, whereas, for the second transition of $\sigma-n$ nature, our TBEs are $2.80\left(\mathrm{~F}_{2} \mathrm{BO}\right)$ and 2.99 $\left(\mathrm{F}_{2} \mathrm{BS}\right) \mathrm{eV}$. For these two compounds, the most advanced 
previous calculations are likely the ROCC3/aug-cc-pVTZ values of 0.71 and $2.78 \mathrm{eV}\left(\mathrm{F}_{2} \mathrm{BO}\right)$, and 0.47 and $2.93 \mathrm{eV}$ $\left(\mathrm{F}_{2} \mathrm{BS}\right)$ obtained by some of us in a recent study. ${ }^{89}$ For the former radical, these values are also very close to earlier CASPT2 $(0.70 \text { and } 2.93 \mathrm{eV})^{165}$ and SAC-CI (0.73 and 2.89 $\mathrm{eV})^{166}$ estimates. The $T_{0}$ energies of these two states were both measured recently as well: 0.65 and $2.78 \mathrm{eV}$ for the oxygen derivative, ${ }^{167}$ and 0.44 and $2.87 \mathrm{eV}$ for the sulfur radical. ${ }^{168}$ These two works and an earlier study by the same group, ${ }^{169}$ also provide advanced theoretical studies of both the $0-0$ transitions and vibronic couplings.

$\mathrm{H}_{2} \mathrm{BO}$. This lighter analogue of $\mathrm{F}_{2} \mathrm{BO}$ remains to be detected experimentally, but its excited states have been studied twice with $a b$ initio theoretical methods, ${ }^{166,169}$ the most recent SAC-CI estimates for the lowest-lying transitions being 2.08 and $3.49 \mathrm{eV}$. ${ }^{166}$ These SAC-CI excitation energies are within $0.10 \mathrm{eV}$ of our FCI-based TBEs.

$\mathrm{HCO}$ and HOC (formyl and isoformyl). For the formyl radical, our TBEs are 2.09 and $5.49 \mathrm{eV}$. Kus and Bartlett reported CCSDT/6-311++G(d,p) transition energies of 2.17 and $5.29 \mathrm{eV}$ (likely the best vertical estimates available previously), ${ }^{170}$ obviously close to ours for the former valence transition. We are also aware of earlier CASPT2 estimates of 2.07 and $5.45 \mathrm{eV}$ for these two states, ${ }^{171}$ that happen to be within $\pm 0.04 \mathrm{eV}$ of our TBEs. There are detailed studies of the potential energy surfaces for the ground and lowest excited states of $\mathrm{HCO} .{ }^{172}$ For isoformyl, the convergence with respect to the basis set is fast and the lowest excited state is well converged with our FCI approach. Hence, we propose a safe TBE of $0.91 \mathrm{eV}$ for the lowest vertical excitation. Most previous studies did not, once more, discuss vertical transition energies. However, we are aware of a recent $0.87 \mathrm{eV} \mathrm{CC}$ estimate for the adiabatic energy obtained with a large basis set. ${ }^{173}$

$\mathrm{H}_{2} \mathrm{PO}$ and $\mathrm{H}_{2} \mathrm{PS}$. These two radical homologues of formaldehyde are puckered in their ground state, and CCSDT is already giving very accurate estimates. Indeed, the CCSDT values are consistent with their FCI counterparts, and one likely needs a triple- $\zeta$ basis set to be close to convergence. The only previous experimental and theoretical studies we are aware of for these two compounds are rather recent. ${ }^{89,174,175}$ They reported: (i) CCSD/aug-cc-pVTZ adiabatic energies of 1.42 and $3.32 \mathrm{eV}$ for $\mathrm{H}_{2} \mathrm{PO},{ }^{174}$ and 0.57 and $2.58 \mathrm{eV}$ for its sulfur counterpart; ${ }^{175}$ (ii), ROCC 3 vertical transitions to the lowest ${ }^{2} A^{\prime}$ states of $4.35 \mathrm{eV}\left(\mathrm{H}_{2} \mathrm{PO}\right)$ and $2.78 \mathrm{eV}\left(\mathrm{H}_{2} \mathrm{PS}\right) .{ }^{89}$ The latter are obviously compatible with the present data.

Nitromethyl. For this (comparatively) large derivative, even the UCCSDT/aug-cc-pVTZ calculations are a challenge in terms of computational resources. The calculations converge too slowly with the number of determinants to ensure valuable FCI extrapolations, except for the second state for which the CCSDT estimate falls within the extrapolation error bar. Fortunately, for all transitions, the difference between ROCC 3 and UCCSDT estimates are small, and we can safely propose our CCSDT values as references. These values of $2.05,2.38,2.56$, and $5.35 \mathrm{eV}$ do agree rather well with the 2005 ROCC3/Sadlej-TZ estimates of 2.03, 2.41, 2.53 and $5.28 \mathrm{eV},{ }^{133}$ that remain the most advances carried out previously to the very best of our knowledge. Retrospectively, the
MRCI excitation energies of 1.25 and $1.52 \mathrm{eV}$ for the two lowest states seem way too low. ${ }^{176}$ The measured photoelectron spectrum of the related anion indicates the presence of the ${ }^{2} A_{2}$ transition at $1.59 \mathrm{eV}$ in the radical, ${ }^{177}$ whereas a rough estimate of $4.25 \mathrm{eV}$ can also be deduced from experimental data for the ${ }^{2} B_{1}$ state. ${ }^{178}$ We trust that the TBEs given in Table 3 are more trustworthy estimates of the vertical transition energies than these indirect experimental transition energies.

$N O$. This highly reactive radical is unsurprisingly quite difficult to capture with theoretical approaches and our current TBEs of 6.12 and $7.21 \mathrm{eV}$ for the two lowest Rydberg states are significantly above the vertical experimental energies of 5.93 and $7.03 \mathrm{eV} .{ }^{139}$ Our geometry is associated with a NO bond distance of $1.149 \AA$ slightly larger than the experimental value of $1.115 \AA$. Moreover, basis set convergence is slow, so that a quadruple- $\zeta$ basis might still be insufficient to be close to the CBS limit for the second excited state.

$\mathrm{OH}$. For $\mathrm{OH}$, the convergence of the CC energy with respect to the excitation degree is extremely fast, but the basis set effects are non-negligible. Our TBEs are 4.09 and 8.11 $\mathrm{eV}$ for the ${ }^{2} \Sigma^{+}$and ${ }^{2} \Sigma^{-}$transitions, respectively. The former value compares very nicely with the experimental one $(4.08 \mathrm{eV}),{ }^{139}$ and is smaller than previous MRCI estimates of $4.27^{179}$ and $4.22 \mathrm{eV} .{ }^{145}$ In contrast, for the ${ }^{2} \Sigma^{-}$transition, our estimate is higher than a previously reported value of 7.87 eV. ${ }^{179}$

Vinyl. For this final radical, we considered four states, two in each spatial symmetry. For the lowest transition of $\pi \rightarrow n$ nature, our FCI/aug-cc-pVTZ result is $3.26 \pm 0.02 \mathrm{eV}$, and one can find many previous calculations yielding similar transition energies: 3.17 (MRCI), ${ }^{180} 3.24$ (MRCI), ${ }^{181} 3.31$ (CCSD), ${ }^{182}$ and $3.30 \mathrm{eV}(\mathrm{CC} 3),{ }^{89}$ whereas the measured 0-0 energy is $2.49 \mathrm{eV} .{ }^{183}$ For the second transition of the same $A^{\prime \prime}$ symmetry and of $n \rightarrow \pi^{\star}$ character, the previous theoretical values we are aware of are $4.78 \mathrm{eV}$ (MRCI) ${ }^{181}$ and $4.93 \mathrm{eV}$ (CCSD). ${ }^{182}$ Our TBE of $4.69 \mathrm{eV}$ is lower. The lowest ${ }^{2} A^{\prime}$ transition is a tricky valence excitation of $\pi \rightarrow \pi^{\star}$ character with a significant multi-excitation character, and we decided to use ROCC for this specific case. It is clear from Table 3 that one needs to go as high as CCSDTQ to be close to FCI. Our TBE of $5.60 \mathrm{eV}$ can be compared to previous estimates of 5.58 $\mathrm{eV}$ (MRCI) ${ }^{181}$ or $5.60 \mathrm{eV}$ (spin-flip CCSD), ${ }^{182}$ which clearly highlights the fantastic accuracy of the spin-flip approach for such transition. Eventually, the last transition of Rydberg character is easier to describe at the CC level, with our TBE of $6.20 \mathrm{eV}$ again close to previously reported results: 6.25 (MRCI) ${ }^{181}$ and $6.31 \mathrm{eV}$ (CCSD). ${ }^{182}$

\subsubsection{Benchmarks}

As for the exotic set, we have used our TBEs/aug-cc-pVTZ to perform benchmarks of "lower-order" methods, and we have especially compared the U and RO versions of CCSD and $\mathrm{CC} 3$, considering all transition energies listed in Table 3 (except three particularly challenging ones that have been omitted, see footnote $g$ in the corresponding Table). The raw data are listed in Table S3 of the SI, whereas Table 4 and Figure 2 gathers the associated statistical data. As expected from previous works, ${ }^{89,133,182}$ the excitation energy errors associated with these doublet-doublet transitions in openshell molecules tend to be larger than for closed-shell systems. 
Indeed, we note that (i) CCSD overshoots by more than $1 \mathrm{eV}$ the transition energies of the second and third excited states of $\mathrm{CH}$; (ii) the MAE obtained with $\mathrm{CC} 3$ is $0.05 \mathrm{eV}$, five times larger than in the exotic set; and (iii) the error dispersion is obviously larger in Figure 2 than in Figure 1. This confirms that accurately describing doublet-doublet transition energies is very challenging. On a more positive note, we observe that the statistical results are improved by using a RO starting point instead of the usual $\mathrm{U}$ approximation, an effect particularly significant at the CCSD level.

Table 4: Statistical values obtained by comparing the results of various methods to the TBE/aug-cc-pVTZ reported in Table S3. See caption of Table 2 for more details.

\begin{tabular}{lccccc}
\hline Method & Count & MSE & MAE & RMSE & SDE \\
\hline UCCSD & 48 & 0.19 & 0.20 & 0.35 & 0.30 \\
ROCCSD & 48 & 0.14 & 0.15 & 0.30 & 0.27 \\
UCC 3 & 48 & 0.03 & 0.06 & 0.11 & 0.11 \\
ROCC 3 & 48 & 0.02 & 0.05 & 0.10 & 0.10
\end{tabular}
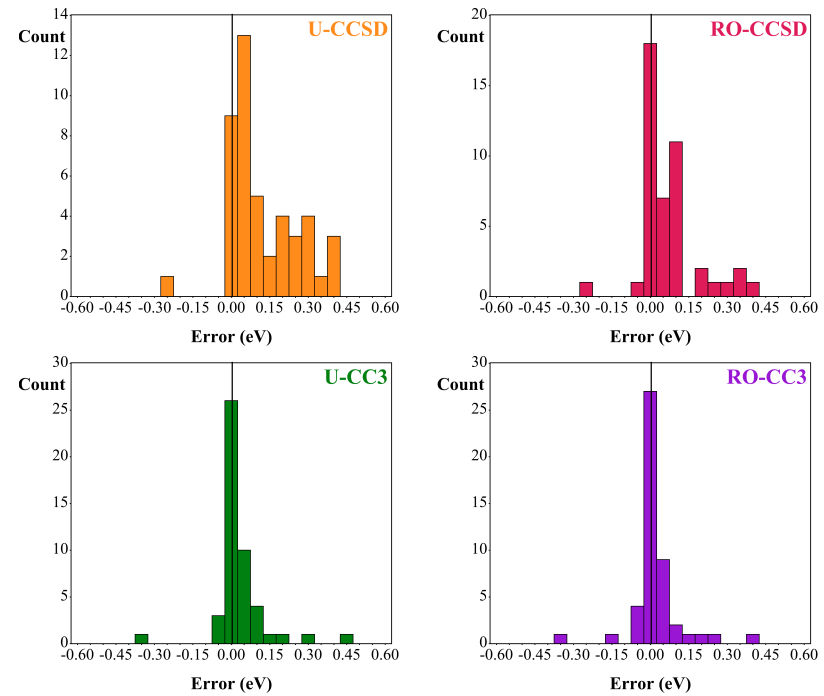

Figure 2: Histograms of the error distribution (in $\mathrm{eV}$ ) obtained with 4 theoretical methods, choosing the TBE/aug-cc-pVTZ of Table 3 as references (raw data in Table S3). For the CCSD cases, even larger errors (out of scale) are observed.

\section{CONCLUSIONS}

In order to complete our three previous sets of highly-accurate excitation energies, ${ }^{23,38,39}$ we have reported here two additional sets of TBEs for: (i) 30 excited states in a series of "exotic" closed-shell compounds including (at least) one of the following atoms: $\mathrm{F}, \mathrm{Cl}, \mathrm{Si}$, or $\mathrm{P}$; (ii) 51 doublet-doublet transitions in a series of radicals characterized by an openshell electronic configuration. In all cases, we have reported at least aug-cc-pVTZ estimates, the vast majority being obtained at the FCI level, and we have applied increasingly accurate $\mathrm{CC}$ methods to ascertain these estimates. For most of these transitions, it is very likely that the present TBEs are the most accurate published to date (for a given geometry).

For the former exotic set, these TBEs have been used to assess the performances of fifteen "lower-order" wave func- tion approaches, including several $\mathrm{CC}$ and $\mathrm{ADC}$ variants. Consistently with our previous works, we found that $\mathrm{CC} 3$ is astonishingly accurate with a MAE as small as $0.01 \mathrm{eV}$ and a SDE of $0.02 \mathrm{eV}$, whereas the trends for the other methods are similar to the one obtained on more standard organic compounds. In contrast, for the radical set, even the refined ROCC 3 method yields a MAE of $0.05 \mathrm{eV}$, and a rather large SDE of $0.10 \mathrm{eV}$. Likewise, the excitation energies obtained with CCSD are much less satisfying for open-shell derivatives (MAE of $0.20 \mathrm{eV}$ with UCCSD and $0.15 \mathrm{eV}$ with ROCCSD) than for the closed-shell systems (MAE of $0.07 \mathrm{eV}$ ).

We hope that these two new sets, which provide a fair ground for the assessments of high-level excited-state models, will be an additional valuable asset for the electronic structure community, and will stimulate further developments in the field.

\section{ACKNOWLEDGEMENTS}

PFL thanks the Centre National de la Recherche Scientifique for funding. This research used resources of (i) the GENCITGCC (Grant No. 2019-A0060801738); (ii) CALMIP under allocation 2020-18005 (Toulouse); (iii) CCIPL (Centre de Calcul Intensif des Pays de Loire); (iv) a local Troy cluster and (v) HPC resources from ArronaxPlus (grant ANR-11-EQPX0004 funded by the French National Agency for Research).

\section{SUPPORTING INFORMATION AVAILABLE}

The Supporting Information is available free of charge at https://pubs.acs.org/doi/10.1021/doi.

Basis set effects at CC 3 level for the exotic set. Benchmark data. Multi-reference values for CON. Cartesian coordinates.

\section{REFERENCES}

(1) Bender, C. F.; Davidson, E. R. Studies in Configuration Interaction: The First-Row Diatomic Hydrides. Phys. Rev. 1969, 183, 23-30.

(2) Whitten, J. L.; Hackmeyer, M. Configuration Interaction Studies of Ground and Excited States of Polyatomic Molecules. I. The CI Formulation and Studies of Formaldehyde. J. Chem. Phys. 1969, 51, 55845596.

(3) Huron, B.; Malrieu, J. P.; Rancurel, P. Iterative Perturbation Calculations of Ground and Excited State Energies from Multiconfigurational Zeroth-Order Wavefunctions. J. Chem. Phys. 1973, 58, 5745-5759.

(4) Giner, E.; Scemama, A.; Caffarel, M. Using Perturbatively Selected Configuration Interaction in Quantum Monte Carlo Calculations. Can. J. Chem. 2013, 91, 879-885.

(5) Caffarel, M.; Giner, E.; Scemama, A.; RamírezSolís, A. Spin Density Distribution in Open-Shell Transition Metal Systems: A Comparative PostHartree-Fock, Density Functional Theory, and Quantum Monte Carlo Study of the $\mathrm{CuCl}_{2}$ Molecule. $J$. Chem. Theory Comput. 2014, 10, 5286-5296.

(6) Evangelista, F. A. Adaptive Multiconfigurational Wave Functions. J. Chem. Phys. 2014, 140, 124114. 
(7) Giner, E.; Scemama, A.; Caffarel, M. Fixed-Node Diffusion Monte Carlo Potential Energy Curve of the Fluorine Molecule $\mathrm{F}_{2}$ Using Selected Configuration Interaction Trial Wavefunctions. J. Chem. Phys. 2015, $142,044115$.

(8) Garniron, Y.; Scemama, A.; Loos, P.-F.; Caffarel, M. Hybrid Stochastic-Deterministic Calculation of the Second-Order Perturbative Contribution of Multireference Perturbation Theory. J. Chem. Phys. 2017, 147, 034101.

(9) Caffarel, M.; Applencourt, T.; Giner, E.; Scemama, A. Toward an Improved Control of the Fixed-Node Error in Quantum Monte Carlo: The Case of the Water Molecule. J. Chem. Phys. 2016, 144, 151103.

(10) Caffarel, M.; Applencourt, T.; Giner, E.; Scemama, A. Recent Progress in Quantum Monte Carlo; 2016; Chapter 2, pp 15-46.

(11) Schriber, J. B.; Evangelista, F. A. An Adaptive Configuration Interaction Approach for Strongly Correlated Electrons with Tunable Accuracy. J. Chem. Phys. 2016, 144, 161106.

(12) Holmes, A. A.; Tubman, N. M.; Umrigar, C. J. HeatBath Configuration Interaction: An Efficient Selected Configuration Interaction Algorithm Inspired by HeatBath Sampling. J. Chem. Theory Comput. 2016, 12, 3674-3680.

(13) Liu, W.; Hoffmann, M. R. iCI: Iterative CI toward full CI. J. Chem. Theory Comput. 2016, 12, 1169-1178.

(14) Sharma, S.; Holmes, A. A.; Jeanmairet, G.; Alavi, A.; Umrigar, C. J. Semistochastic Heat-Bath Configuration Interaction Method: Selected Configuration Interaction with Semistochastic Perturbation Theory. $J$. Chem. Theory Comput. 2017, 13, 1595-1604.

(15) Holmes, A. A.; Umrigar, C. J.; Sharma, S. Excited States Using Semistochastic Heat-Bath Configuration Interaction. J. Chem. Phys. 2017, 147, 164111.

(16) Chien, A. D.; Holmes, A. A.; Otten, M.; Umrigar, C. J.; Sharma, S.; Zimmerman, P. M. Excited States of Methylene, Polyenes, and Ozone from HeatBath Configuration Interaction. J. Phys. Chem. A 2018, 122, 2714-2722.

(17) Garniron, Y.; Scemama, A.; Giner, E.; Caffarel, M.; Loos, P.-F. Selected Configuration Interaction Dressed by Perturbation. J. Chem. Phys. 2018, 149, 064103.

(18) Scemama, A.; Garniron, Y.; Caffarel, M.; Loos, P. F. Deterministic Construction of Nodal Surfaces Within Quantum Monte Carlo: The Case of FeS. J. Chem. Theory Comput. 2018, 14, 1395-1402.

(19) Garniron, Y.; Applencourt, T.; Gasperich, K.; Benali, A.; Ferté, A.; Paquier, J.; Pradines, B.; Assaraf, R.; Reinhardt, P.; Toulouse, J.; Barbaresco, P.; Renon, N.; David, G.; Malrieu, J.-P.; Véril, M.; Caffarel, M.; Loos, P.-F.; Giner, E.; Scemama, A. Quantum Package 2.0: An Open-Source DeterminantDriven Suite of Programs. J. Chem. Theory Comput. 2019, 15, 3591-3609.

(20) Loos, P.-F.; Scemama, A.; Blondel, A.; Garniron, Y.; Caffarel, M.; Jacquemin, D. A Mountaineering Strategy to Excited States: Highly-Accurate Reference En- ergies and Benchmarks. J. Chem. Theory Comput. 2018, 14, 4360-4379.

(21) Loos, P.-F.; Boggio-Pasqua, M.; Scemama, A.; Caffarel, M.; Jacquemin, D. Reference Energies for Double Excitations. J. Chem. Theory Comput. 2019, 15, 1939-1956.

(22) Giner, E.; Scemama, A.; Toulouse, J.; Loos, P. F. Chemically Accurate Excitation Energies With Small Basis Sets. J. Chem. Phys. 2019, 151, 144118.

(23) Loos, P.-F.; Lipparini, F.; Boggio-Pasqua, M.; Scemama, A.; Jacquemin, D. A Mountaineering Strategy to Excited States: Highly-Accurate Energies and Benchmarks for Medium Size Molecules. J. Chem. Theory Comput. 2020, 16, 1711-1741.

(24) Loos, P. F.; Scemama, A.; Jacquemin, D. The Quest for Highly-Accurate Excitation Energies: A Computational Perspective. J. Phys. Chem. Lett. 2020, in press, doi: 10.1021/acs.jpclett.0c00014.

(25) Kucharski, S. A.; Bartlett, R. J. Recursive Intermediate Factorization and Complete Computational Linearization of the Coupled-Cluster Single, Double, Triple, and Quadruple Excitation Equations. Theor. Chim. Acta 1991, 80, 387-405.

(26) Christiansen, O.; Koch, H.; Jørgensen, P. Response Functions in the CC3 Iterative Triple Excitation Model. J. Chem. Phys. 1995, 103, 7429-7441.

(27) Koch, H.; Christiansen, O.; Jorgensen, P.; Sanchez de Merás, A. M.; Helgaker, T. The CC3 Model: An Iterative Coupled Cluster Approach Including Connected Triples. J. Chem. Phys. 1997, 106, 1808-1818.

(28) Watts, J. D.; Bartlett, R. J. Iterative and Non-Iterative Triple Excitation Corrections in Coupled-Cluster Methods for Excited Electronic States: the EOMCCSDT-3 and EOM-CCSD $(\tilde{T})$ Methods. Chem. Phys. Lett. 1996, 258, 581-588.

(29) Prochnow, E.; Harding, M. E.; Gauss, J. Parallel Calculation of CCSDT and Mk-MRCCSDT Energies. $J$. Chem. Theory Comput. 2010, 6, 2339-2347.

(30) Noga, J.; Bartlett, R. J. The Full CCSDT Model for Molecular Electronic Structure. J. Chem. Phys. 1987, 86, 7041-7050.

(31) Roos, B. O.; Andersson, K.; Fulscher, M. P.; Malmqvist, P.-A.; Serrano-Andrés, L. In Adv. Chem. Phys.; Prigogine, I., Rice, S. A., Eds.; Wiley, New York, 1996; Vol. XCIII; pp 219-331.

(32) Andersson, K.; Malmqvist, P. A.; Roos, B. O.; Sadlej, A. J.; Wolinski, K. Second-Order Perturbation Theory With a CASSCF Reference Function. $J$. Phys. Chem. 1990, 94, 5483-5488.

(33) Angeli, C.; Cimiraglia, R.; Evangelisti, S.; Leininger, T.; Malrieu, J.-P. Introduction of $n$ Electron Valence States for Multireference Perturbation Theory. J. Chem. Phys. 2001, 114, 10252-10264.

(34) Angeli, C.; Cimiraglia, R.; Malrieu, J.-P. N-Electron Valence State Perturbation Theory: A Fast Implementation of the Strongly Contracted Variant. Chem. Phys. Lett. 2001, 350, 297-305.

(35) Angeli, C.; Cimiraglia, R.; Malrieu, J.-P. $N$-Electron Valence State Perturbation Theory: A Spinless For- 
mulation and an Efficient Implementation of the Strongly Contracted and of the Partially Contracted Variants. J. Chem. Phys. 2002, 117, 9138-9153.

(36) Kozma, B.; Tajti, A.; Demoulin, B.; Izsak, R.; Nooijen, M.; Szalay, P. G. A New Benchmark Set for Excitation Energy of Charge Transfer States: Systematic Investigation of Coupled-Cluster Type Methods. ChemRxiv 2020, 10.26434/chemrxiv.11858010.v1.

(37) Hahn, D. K.; RaghuVeer, K.; Ortiz, J. V. Computational Tests of Quantum Chemical Models for Excited and Ionized States of Molecules with Phosphorus and Sulfur Atoms. J. Phys. Chem. A 2014, 118, 35143524.

(38) Loos, P.-F.; Galland, N.; Jacquemin, D. Theoretical 00 Energies with Chemical Accuracy. J. Phys. Chem. Lett. 2018, 9, 4646-4651.

(39) Loos, P.-F.; Jacquemin, D. Evaluating 0-0 Energies with Theoretical Tools: a Short Review. ChemPhotoChem 2019, 3, 684-696.

(40) Budzák, Š.; Scalmani, G.; Jacquemin, D. Accurate Excited-State Geometries: a CASPT2 and CoupledCluster Reference Database for Small Molecules. J. Chem. Theory Comput. 2017, 13, 6237-6252.

(41) Jacquemin, D. What is the Key for Accurate Absorption and Emission Calculations? Energy or Geometry ? J. Chem. Theory Comput. 2018, 14, 1534-1543.

(42) Bremond, E.; Savarese, M.; Adamo, C.; Jacquemin, D. Accuracy of TD-DFT Geometries: a Fresh Look. $J$. Chem. Theory Comput. 2018, 14, 3715-3727.

(43) Aidas, K.; Angeli, C.; Bak, K. L.; Bakken, V.; Bast, R.; Boman, L.; Christiansen, O.; Cimiraglia, R.; Coriani, S.; Dahle, P.; Dalskov, E. K.; Ekström, U.; Enevoldsen, T.; Eriksen, J. J.; Ettenhuber, P.; Fernández, B.; Ferrighi, L.; Fliegl, H.; Frediani, L.; Hald, K.; Halkier, A.; Hättig, C.; Heiberg, H.; Helgaker, T.; Hennum, A. C.; Hettema, H.; Hjertenæs, E.; Høst, S.; Hoyvik, I.-M.; Iozzi, M. F.; Jansik, B.; Jensen, H. J. A.; Jonsson, D.; Jørgensen, P.; Kauczor, J.; Kirpekar, S.; Kjærgaard, T.; Klopper, W.; Knecht, S.; Kobayashi, R.; Koch, H.; Kongsted, J.; Krapp, A.; Kristensen, K.; Ligabue, A.; Lutnæs, O. B.; Melo, J. I.; Mikkelsen, K. V.; Myhre, R. H.; Neiss, C.; Nielsen, C. B.; Norman, P.; Olsen, J.; Olsen, J. M. H.; Osted, A.; Packer, M. J.; Pawlowski, F.; Pedersen, T. B.; Provasi, P. F.; Reine, S.; Rinkevicius, Z.; Ruden, T. A.; Ruud, K.; Rybkin, V. V.; Sałek, P.; Samson, C. C. M.; de Merás, A. S.; Saue, T.; Sauer, S. P. A.; Schimmelpfennig, B.; Sneskov, K.; Steindal, A. H.; Sylvester-Hvid, K. O.; Taylor, P. R.; Teale, A. M.; Tellgren, E. I.; Tew, D. P.; Thorvaldsen, A. J.; Thogersen, L.; Vahtras, O.; Watson, M. A.; Wilson, D. J. D.; Ziolkowski, M.; Ågren, H. The Dalton Quantum Chemistry Program System. WIREs Comput. Mol. Sci. 2014, 4, 269-284.

(44) CFOUR, Coupled-Cluster techniques for Computational Chemistry, a quantum-chemical program package by J.F. Stanton, J. Gauss, L. Cheng, M.E. Harding, D.A. Matthews, P.G. Szalay with contributions from A.A. Auer, R.J. Bartlett, U. Benedikt, C. Berger,
D.E. Bernholdt, Y.J. Bomble, O. Christiansen, F. Engel, R. Faber, M. Heckert, O. Heun, M. Hilgenberg, C. Huber, T.-C. Jagau, D. Jonsson, J. Jusélius, T. Kirsch, K. Klein, W.J. Lauderdale, F. Lipparini, T. Metzroth, L.A. Mück, D.P. O'Neill, D.R. Price, E. Prochnow, C. Puzzarini, K. Ruud, F. Schiffmann, W. Schwalbach, C. Simmons, S. Stopkowicz, A. Tajti, J. Vázquez, F. Wang, J.D. Watts and the integral packages MOLECULE (J. Almlöf and P.R. Taylor), PROPS (P.R. Taylor), ABACUS (T. Helgaker, H.J. Aa. Jensen, P. Jørgensen, and J. Olsen), and ECP routines by A. V. Mitin and C. van Wüllen. For the current version, see http://www.cfour.de.

(45) Frisch, M. J.; Trucks, G. W.; Schlegel, H. B.; Scuseria, G. E.; Robb, M. A.; Cheeseman, J. R.; Scalmani, G.; Barone, V.; Petersson, G. A.; Nakatsuji, H.; Li, X.; Caricato, M.; Marenich, A. V.; Bloino, J.; Janesko, B. G.; Gomperts, R.; Mennucci, B.; Hratchian, H. P.; Ortiz, J. V.; Izmaylov, A. F.; Sonnenberg, J. L.; Williams-Young, D.; Ding, F.; Lipparini, F.; Egidi, F.; Goings, J.; Peng, B.; Petrone, A.; Henderson, T.; Ranasinghe, D.; Zakrzewski, V. G.; Gao, J.; Rega, N.; Zheng, G.; Liang, W.; Hada, M.; Ehara, M.; Toyota, K.; Fukuda, R.; Hasegawa, J.; Ishida, M.; Nakajima, T.; Honda, Y.; Kitao, O.; Nakai, H.; Vreven, T.; Throssell, K.; Montgomery, J. A., Jr.; Peralta, J. E.; Ogliaro, F.; Bearpark, M. J.; Heyd, J. J.; Brothers, E. N.; Kudin, K. N.; Staroverov, V. N.; Keith, T. A.; Kobayashi, R.; Normand, J.; Raghavachari, K.; Rendell, A. P.; Burant, J. C.; Iyengar, S. S.; Tomasi, J.; Cossi, M.; Millam, J. M.; Klene, M.; Adamo, C.; Cammi, R.; Ochterski, J. W.; Martin, R. L.; Morokuma, K.; Farkas, O.; Foresman, J. B.; Fox, D. J. Gaussian 16 Revision A.03. 2016; Gaussian Inc. Wallingford CT.

(46) Rolik, Z.; Szegedy, L.; Ladjánszki, I.; Ladóczki, B.; Kállay, M. An Efficient Linear-Scaling CCSD(T) Method Based on Local Natural Orbitals. J. Chem. Phys. 2013, 139, 094105.

(47) Kállay, M.; Rolik, Z.; Csontos, J.; Nagy, P.; Samu, G.; Mester, D.; Csóka, J.; Szabó, B.; Ladjánszki, I.; Szegedy, L.; Ladóczki, B.; Petrov, K.; Farkas, M.; Mezei, P. D.; Hégely., B. MRCC, Quantum Chemical Program. 2017; See: www.mrcc.hu.

(48) Prascjer, B. P.; Woon, D. E.; Peterson, K. A.; Dunning, T. H.; Wilson, A. K. Gaussian Basis Sets for use in Correlated Molecular Calculations. VII. Valence, Core-valence, and Scalar Relativistic Basis Sets for $\mathrm{Li}, \mathrm{Be}, \mathrm{Na}$, and Mg. Theor. Chem. Acc. 2010, 128, 69-82.

(49) Scemama, A.; Caffarel, M.; Benali, A.; Jacquemin, D.; Loos, P.-F. Influence of Pseudopotentials on Excitation Energies from Selected Configuration Interaction and Diffusion Monte Carlo. Res. Chem. 2019, 1,100002 .

(50) TURBOMOLE V7.3 2018, a development of University of Karlsruhe and Forschungszentrum Karlsruhe GmbH, 1989-2007, TURBOMOLE GmbH, since 
2007; available from http://www. turbomole.com (accessed 13 June 2016).

(51) Head-Gordon, M.; Rico, R. J.; Oumi, M.; Lee, T. J. A Doubles Correction to Electronic Excited States From Configuration Interaction in the Space of Single Substitutions. Chem. Phys. Lett. 1994, 219, 21-29.

(52) Head-Gordon, M.; Maurice, D.; Oumi, M. A Perturbative Correction to Restricted Open-Shell Configuration-Interaction with Single Substitutions for Excited-States of Radicals. Chem. Phys. Lett. 1995, 246, 114-121.

(53) Krylov, A. I.; Gill, P. M. Q-Chem: an Engine for Innovation. WIREs Comput. Mol. Sci. 2013, 3, 317326.

(54) Stanton, J. F.; Gauss, J. Perturbative Treatment of the Similarity Transformed Hamiltonian in Equation-ofMotion Coupled-Cluster Approximations. J. Chem. Phys. 1995, 103, 1064-1076.

(55) Trofimov, A. B.; Stelter, G.; Schirmer, J. Electron Excitation Energies Using a Consistent Third-Order Propagator Approach: Comparison with Full Configuration Interaction and Coupled Cluster Results. $J$. Chem. Phys. 2002, 117, 6402-6410.

(56) Harbach, P. H. P.; Wormit, M.; Dreuw, A. The Third-Order Algebraic Diagrammatic Construction Method (ADC(3)) for the Polarization Propagator for Closed-Shell Molecules: Efficient Implementation and Benchmarking. J. Chem. Phys. 2014, 141, 064113.

(57) Dreuw, A.; Wormit, M. The Algebraic Diagrammatic Construction Scheme for the Polarization Propagator for the Calculation of Excited States. WIREs Comput. Mol. Sci. 2015, 5, 82-95.

(58) Trofimov, A.; Schirmer, J. Polarization Propagator Study of Electronic Excitation in key Heterocyclic Molecules I. Pyrrole. Chem. Phys. 1997, 214, 153170.

(59) Christiansen, O.; Koch, H.; Jørgensen, P. The SecondOrder Approximate Coupled Cluster Singles and Doubles Model CC2. Chem. Phys. Lett. 1995, 243, 409418.

(60) Hättig, C.; Weigend, F. CC2 Excitation Energy Calculations on Large Molecules Using the Resolution of the Identity Approximation. J. Chem. Phys. 2000, 113, 5154-5161.

(61) Purvis III, G. P.; Bartlett, R. J. A Full Coupled-Cluster Singles and Doubles Model: The Inclusion of Disconnected Triples. J. Chem. Phys. 1982, 76, 1910-1918.

(62) Christiansen, O.; Koch, H.; Jørgensen, P. Perturbative Triple Excitation Corrections to Coupled Cluster Singles and Doubles Excitation Energies. J. Chem. Phys. 1996, 105, 1451-1459.

(63) Neese, F. The ORCA Program System. WIREs Comput. Mol. Sci. 2012, 2, 73-78.

(64) Nooijen, M.; Bartlett, R. J. A New Method for Excited States: Similarity Transformed Equation-Of-Motion Coupled-Cluster Theory. J. Chem. Phys. 1997, 106, 6441-6448.
(65) Dutta, A. K.; Nooijen, M.; Neese, F.; Izsák, R. Exploring the Accuracy of a Low Scaling Similarity Transformed Equation of Motion Method for Vertical Excitation Energies. J. Chem. Theory Comput. 2018, 14, 72-91.

(66) Krauter, C. M.; Pernpointner, M.; Dreuw, A. Application of the Scaled-Opposite-Spin Approximation to Algebraic Diagrammatic Construction Schemes of Second Order. J. Chem. Phys. 2013, 138, 044107.

(67) Hellweg, A.; Grün, S. A.; Hättig, C. Benchmarking the Performance of Spin-Component Scaled CC2 in Ground and Electronically Excited States. Phys. Chem. Chem. Phys. 2008, 10, 4119-4127.

(68) Goerigk, L.; Grimme, S. Assessment of TD-DFT Methods and of Various Spin Scaled $\mathrm{CIS}_{n} \mathrm{D}$ and CC2 Versions for the Treatment of Low-Lying Valence Excitations of Large Organic Dyes. J. Chem. Phys. 2010, $132,184103$.

(69) Parrish, R. M.; Burns, L. A.; Smith, D. G. A.; Simmonett, A. C.; DePrince, A. E.; Hohenstein, E. G.; Bozkaya, U.; Sokolov, A. Y.; Di Remigio, R.; Richard, R. M.; Gonthier, J. F.; James, A. M.; McAlexander, H. R.; Kumar, A.; Saitow, M.; Wang, X.; Pritchard, B. P.; Verma, P.; Schaefer, H. F.; Patkowski, K.; King, R. A.; Valeev, E. F.; Evangelista, F. A.; Turney, J. M.; Crawford, T. D.; Sherrill, C. D. Psi4 1.1: An Open-Source Electronic Structure Program Emphasizing Automation, Advanced Libraries, and Interoperability. J. Chem. Theory Comput. 2017, 13, 3185-3197.

(70) Schreiber, M.; Silva-Junior, M. R.; Sauer, S. P. A.; Thiel, W. Benchmarks for Electronically Excited States: CASPT2, CC2, CCSD and CC3. J. Chem. Phys. 2008, 128, 134110.

(71) Lavín, C.; Velasco, A. M.; Martín, I.; PitarchRuíz, J. V.; Sánchez de Merás, A. M. J.; SánchezMarín, J. Electronic Spectrum of $\mathrm{F}_{2} \mathrm{CO}$ : Theoretical Calculations of Vertical Excitation Energies and Intensities. Theor. Chem. Acc. 2011, 129, 53-61.

(72) Kato, H.; Nunes, Y.; Duflot, D.; Limão-Vieira, P.; Tanaka, H. Electronic States of $\mathrm{F}_{2} \mathrm{CO}$ as Studied by Electron Energy-Loss Spectroscopy and ab Initio Calculations. J. Phys. Chem. A 2011, 115, 2708-2718.

(73) Workman, G. L.; Duncan, A. B. F. Electronic Spectrum of Carbonyl Fluoride. J. Chem. Phys. 1970, 52, 3204-3209.

(74) Judge, R. H.; Moule, D. C. Analysis of the $254.7 \mathrm{~nm}$ Absorption System of Carbonyl Fluoride. J. Chem. Phys. 1983, 78, 4806-4810.

(75) Bokarev, S. I.; Dolgov, E. K.; Bataev, V. A.; Godunov, I. A. Molecular Parameters of Tetraatomic Carbonyls $\mathrm{X}_{2} \mathrm{CO}$ and $\mathrm{XYCO}(\mathrm{X}, \mathrm{Y}=\mathrm{H}, \mathrm{F}, \mathrm{Cl})$ in the Ground and Lowest Excited Electronic States, Part 1: A Test of Ab Initio Methods. Int. J. Quantum Chem. 2009, 109, 569-585.

(76) Czernek, J.; Zivny, O. The EOM-CC Studies of LowLying Electronic States of $\mathrm{NO}_{2}^{-}, \mathrm{CCl}_{2}$ and $\mathrm{OF}_{2}^{+}$. Chem. Phys. Lett. 2007, 435, 29-33. 
(77) Cai, Z.-L.; Zhang, X.-G.; Wang, X.-Y. Ab initio Study of the Electronic Spectrum of Dichlorocarbene $\mathrm{CCl}_{2}$. Chem. Phys. Lett. 1993, 210, 481-487.

(78) Sun, E.; Ren, T.; Shan, S.; Liu, Q.; Xu, H.; Yan, B. Multireference Configuration Interaction Study of Dichlorocarbene. Chem. Phys. 2015, 459, 54-58.

(79) Sun, E.-P.; Liu, Q.-X.; Ren, T.-Q.; Shan, S.-M.; $\mathrm{Xu}$, H.-F.; Yan, B. Examination of Potential Energy Curves of $\mathrm{CFCl}$ by Multi-reference Configuration Interaction Method. Chin. Chem. Lett. 2015, 32, 123101.

(80) Schirmer, J. Closed-Form Intermediate Representations of Many-Body Propagators and Resolvent Matrices. Phys. Rev. A. 1991, 43, 4647-4659.

(81) Karolczak, J.; Joo, D. L.; Clouthier, D. J. The Electronic Spectrum of Chlorofluorocarbene. J. Chem. Phys. 1993, 99, 1447-1456.

(82) Guss, J. S.; Votava, O.; Kable, S. H. Electronic Spectroscopy of Jet-Cooled CFCl: Laser-Induced Fluorescence, Dispersed Fluorescence, Lifetimes, and CCl Dissociation Barrier. J. Chem. Phys. 2001, 115, 11118-11130.

(83) Er-Ping, S.; Ting-Qi, R.; Qi-Xin, L.; Quan, M.; JinJuan, Z.; Hai-Feng, X.; Bing, Y. Electronic States of Difluorocarbene Calculated by Multireference Configuration Interaction Method. Chin. Chem. Lett. 2016, 33, 023101.

(84) Pandey, R. R.; Khait, Y. G.; Hoffmann, M. R. Ground and Low-Lying Excited Electronic States of Difluorodiazirine. J. Phys. Chem. A 2004, 108, 3119-3124.

(85) Lombardi, J. R.; Klemperer, W.; Robin, M. B.; Basch, H.; Kuebler, N. A. Optical Spectra of Small Rings. I. The $n \rightarrow \pi^{\star}$ Transition of Difluorodiazirine. J. Chem. Phys. 1969, 51, 33-44.

(86) Hepburn, P.; Hollas, J. The 352 nm Absorption Spectrum of Difluorodiazirine. J. Mol. Spectrosc. 1974, 50, 126-141.

(87) Sieber, H.; Riedle, E.; Neusser, H. Doppler-Free TwoPhoton Spectrum of the $0_{0}^{0}$ Band of the $\tilde{A}^{1} B_{1} \leftarrow \tilde{X}^{1} A_{1}$ Transition in Difluorodiazirine, $\mathrm{F}_{2} \mathrm{CN}_{2}$. Chem. Phys. Lett. 1990, 169, 191-197.

(88) Terrabuio, L. A.; Haiduke, R. L. A.; Matta, C. F. Difluorodiazirine $\left(\mathrm{CF}_{2} \mathrm{~N}_{2}\right)$ : A Comparative Quantum Mechanical Study of the First Triplet and First Singlet Excited States. Chem. Phys. Lett. 2016, 655-656, 96102.

(89) Loos, P.-F.; Jacquemin, D. Chemically Accurate 0-0 Energies with not-so-Accurate Excited State Geometries. J. Chem. Theory Comput. 2019, 15, 2481-2491.

(90) Giddings, L.; Innes, K. The Electronic Spectra and Vibrational Assignments of Carbonyl Chloride and Formyl Fluoride. J. Mol. Spectrosc. 1962, 8, 328-337.

(91) Fischer, G. The 2670 Absorption System of Formyl Fluoride. J. Mol. Spectrosc. 1969, 29, 37-53.

(92) Stanton, J. F.; Gauss, J. Analytic Energy Derivatives for the Equation-of-Motion Coupled-Cluster Method: Algebraic Expressions, Implementation and Application to the $S_{1}$ State of HFCO. Theor. Chim. Acta 1995, 91, 267-289.
(93) Crane, J. C.; Nam, H.; Beal, H. P.; Clauberg, H.; Choi, Y. S.; Moore, C.; Stanton, J. F. Vibrational Assignment of the $S_{1}$ Fluorescence Excitation Spectrum of Formyl Fluoride. J. Mol. Spectrosc. 1997, 181, 5666.

(94) Fang, W.-H.; Liu, R.-Z. Ab initio Studies of Dissociation Pathways on the Ground- and Excited-State Potential Energy Surfaces for HFCO. J. Chem. Phys. 2001, 115, 5411-5417.

(95) Pradhan, E.; Brown, A. The Lowest Lying Excited Electronic States for HFCO Including a Potential Energy Surface for $S_{1}$ in Sum-of-Products Form. Mol. Phys. 2019, doi: 10.1080/00268976.2019.1674936.

(96) Shan, S.; Zhang, X.; Sun, E.; Xu, H.; Yan, B. Theoretical Study on the Excited Electronic States of $\mathrm{CHCl}$ : Application to Photodissociation at $193 \mathrm{~nm}$. J. Phys. Chem. A 2015, 119, 10309-10315.

(97) Christiansen, O.; Ruden, T. A.; Ruud, K.; Helgaker, T. Vibronic Transitions from Coupled-Cluster Response Theory: Theory and Application to $\mathrm{HSiF}$ and $\mathrm{H} 2 \mathrm{O}$. J. Chem. Phys. 2002, 116, 8334-8342.

(98) Ehara, M.; Oyagi, F.; Abe, Y.; Fukuda, R.; Nakatsuji, H. Excited-State Geometries and Vibrational Frequencies Studied Using the Analytical Energy Gradients of the Direct Symmetry-Adapted Cluster-Configuration Interaction Method. I. HAXType Molecules. J. Chem. Phys. 2011, 135, 044316.

(99) Chang, B.-C.; Sears, T. J. Frequency-Modulation Transient Absorption Spectrum of the $\mathrm{HCCl}$ $\tilde{A}^{1} A^{\prime \prime}(0,0,0) \leftarrow \tilde{X}^{1} A^{\prime}(0,0,0)$ Transition. J. Chem. Phys. 1995, 102, 6347-6353.

(100) Kakimoto, M.; Saito, S.; Hirota, E. Doppler-Limited Dye Laser Excitation Spectroscopy of HCF. J. Mol. Spectrosc. 1981, 88, 300-310.

(101) Schmidt, K.; Springborg, M. Static hyperpolarizabilities of infinite conjufated polymers in the framework of density-functional theory. Phys. Chem. Chem. Phys. 1999, 1, 1743-1748.

(102) Harper, W. W.; Karolczak, J.; Clouthier, D. J.; Ross, S. C. Chemical reaction jet spectroscopy, molecular structure, and the bending potential of the $\tilde{A}^{1} A^{\prime}$ state of monofluorosilylene (HSiF). J. Chem. Phys. 1995, 103, 883-891.

(103) Nanbu, S.; Gray, S. K.; Kinoshita, T.; Aoyagi, M. Theoretical Study of the Potential Energy Surfaces and Bound States of HCP. J. Chem. Phys. 2000, 112, 5866-5876.

(104) Ingrosso, F.; Ladanyi, B. M.; Mennucci, B.; Scalmani, G. Solvation of coumarin 153 in supercritical fluoroform. J. Phys. Chem. B 2006, 110, 4953-4962.

(105) Goldstein, E.; Jin, S.; Carrillo, M. R.; Cave, R. J. $\mathrm{Ab}$ Initio Study of the Ground and Excited States of HCP and its Isomer HPC. J. Comput. Chem. 1993, 14, 186-194.

(106) Herzberg, G. Molecular Spectra and Molecular Structure. III. Electronic Spectra and Electronic Structure of Polyatomic Molecules; D. Van Nostrand Company: London, UK, 1966. 
(107) Luna, A.; Merchán, M.; Ross, B. O. A Theoretical Analysis of the Lowest Excited States in $\mathrm{HNO} / \mathrm{NOH}$ and HPO/POH. Chem. Phys. 1995, 196, 437-445.

(108) Tackett, B. S.; Clouthier, D. J. HPO Does not Follow Walsh's rules! Improved Molecular Structures from the Spectroscopy of Jet-Cooled HPO and DPO. J. Chem. Phys. 2002, 117, 10604-10612.

(109) Lee, E. P. F.; Mok, D. K. W.; Chau, F.-T.; Dyke, J. M. Ab Initio Calculations on the $\tilde{X}^{1} A^{\prime}$ and $\tilde{A}^{1} A$ " States of HPO and Franck-Condon Simulation of the Single Vibronic Level Emission Spectraof HPO and DPO. J. Chem. Phys. 2007, 127, 214305.

(110) Grimminger, R.; Clouthier, D. J.; Tarroni, R.; Wang, Z.; Sears, T. J. An Experimental and Theoretical Study of the Electronic Spectrum of HPS, a Second row HNO Analog. J. Chem. Phys. 2013, 139, 174306

(111) Mok, D. K. W.; Lee, E. P. F.; Chau, F.-t.; Dyke, J. M. Simulation of the Single-Vibronic-Level Emission Spectrum of HPS. J. Chem. Phys. 2014, 140, 194311.

(112) Mehnen, B.; Linguerri, R.; Ben Yaghlane, S.; Mogren Al Mogren, M.; Elmarghany, A.; Hochlaf, M. Spectroscopy of the Electronic Excited States of Thioxophosphane, HPS, and of its Deuterated Species. $J$. Chem. Phys. 2018, 149, 164303.

(113) Chau, F.-T.; Wang, D.-C.; Lee, E. P. F.; Dyke, J. M.; Mok, D. K. W. $\tilde{A}^{1} A_{1}, \tilde{a}^{3} B_{1}$, and $\tilde{A}^{1} B_{1}$ States of $\mathrm{SiCl}_{2}$ : $\mathrm{Ab}$ Initio Calculations and Simulation of Emission Spectra. J. Phys. Chem. A 1999, 103, 4925-4932.

(114) Du, K.; Chen, X.; Setser, D. Identification of the $\mathrm{SiCl}_{2}$ $\left(\tilde{a}^{3} B_{1}-\tilde{X}^{1} A_{1}\right)$ Emission System and a Flow Reactor Source of $\mathrm{SiCl}_{2}\left(\tilde{a}^{3} B_{1}\right)$. Chem. Phys. Lett. 1991, 181, 344-350.

(115) Karolczak, J.; Clouthier, D. J. Pyrolysis Jet Spectroscopy of Dichlorosilylene. Chem. Phys. Lett. 1993, 201, 409-415.

(116) Ran, Y.; Pang, M.; Shen, W.; Li, M.; He, R. Vibrationally High-Resolved Electronic Spectra of $\mathrm{MCl}_{2}$ $(\mathrm{M}=\mathrm{C}, \mathrm{Si}, \mathrm{Ge}, \mathrm{Sn}, \mathrm{Pb})$ and Photoelectron Spectra of $\mathrm{MCl}_{2}^{-}$. SpectroChim. Acta A 2016, 167, 1-11.

(117) Kánnár, D.; Tajti, A.; Szalay, P. G. Accuracy of Coupled Cluster Excitation Energies in Diffuse Basis Sets. J. Chem. Theory Comput. 2017, 13, 202-209.

(118) Smith, T. C.; Evans, C. J.; Clouthier, D. J. Discovery of the Optically Forbidden $S_{1}-S_{0}$ Transition of Silylidene $\left(\mathrm{H}_{2} \mathrm{C}=\mathrm{Si}\right)$. J. Chem. Phys. 2003, 118, 1642-1648.

(119) Harper, W. W.; Waddell, K. W.; Clouthier, D. J. Jet Spectroscopy, Structure, Anomalous Fluorescence, and Molecular Quantum Beats of Silylidene $\left(\mathrm{H}_{2} \mathrm{C}=\mathrm{Si}\right)$, the Simplest Unsaturated Silylene. J. Chem. Phys. 1997, 107, 8829-8839.

(120) Hilliard, R. K.; Grev, R. S. The Excited Electronic States of $\mathrm{H}_{2}$ CSi. J. Chem. Phys. 1997, 107, 88238828.

(121) Loos, P.-F.; Jacquemin, D. Is ADC(3) as Accurate as CC3 for Valence and Rydberg Transition Energies? $J$. Phys. Chem. Lett. 2020, 11, 974-980.
(122) Kánnár, D.; Szalay, P. G. Benchmarking Coupled Cluster Methods on Valence Singlet Excited States. J. Chem. Theory Comput. 2014, 10, 3757-3765.

(123) Tajti, A.; Szalay, P. G. Investigation of the Impact of Different Terms in the Second Order Hamiltonian on Excitation Energies of Valence and Rydberg States. $J$. Chem. Theory Comput. 2016, 12, 5477-5482.

(124) Tajti, A.; Tulipán, L.; Szalay, P. G. Accuracy of SpinComponent Scaled ADC(2) Excitation Energies and Potential Energy Surfaces. J. Chem. Theory Comput. 2020, 16, 468-474, PMID: 31820977.

(125) Hättig, C. In Response Theory and Molecular Properties (A Tribute to Jan Linderberg and Poul Jorgensen); Jensen, H. A., Ed.; Advances in Quantum Chemistry; Academic Press, 2005; Vol. 50; pp 37-60.

(126) Sauer, S. P. A.; Schreiber, M.; Silva-Junior, M. R.; Thiel, W. Benchmarks for Electronically Excited States: A Comparison of Noniterative and Iterative Triples Corrections in Linear Response Coupled Cluster Methods: CCSDR(3) versus CC3.J. Chem. Theory Comput. 2009, 5, 555-564.

(127) Watson, T. J.; Lotrich, V. F.; Szalay, P. G.; Perera, A.; Bartlett, R. J. Benchmarking for Perturbative TripleExcitations in EE-EOM-CC Methods. J. Phys. Chem. A 2013, 117, 2569-2579.

(128) Suellen, C.; Garcia Freitas, R.; Loos, P.-F.; Jacquemin, D. Cross Comparisons Between Experiment, TD-DFT, CC, and ADC for Transition Energies. J. Chem. Theory Comput. 2019, 15, 4581-4590.

(129) Caricato, M.; Trucks, G. W.; Frisch, M. J.; Wiberg, K. B. Electronic Transition Energies: A Study of the Performance of a Large Range of Single Reference Density Functional and Wave Function Methods on Valence and Rydberg States Compared to Experiment. J. Chem. Theory Comput. 2010, 6, 370-383.

(130) Jacquemin, D.; Duchemin, I.; Blase, X. Is the BetheSalpeter Formalism Accurate for Excitation Energies? Comparisons with TD-DFT, CASPT2, and EOMCCSD. J. Phys. Chem. Lett. 2017, 8, 1524-1529.

(131) Jacquemin, D.; Duchemin, I.; Blase, X. 0-0 Energies Using Hybrid Schemes: Benchmarks of TD-DFT, CIS(D), ADC(2), CC2, and BSE/GW formalisms for 80 Real-Life Compounds. J. Chem. Theory Comput. 2015, 11, 5340-5359.

(132) Winter, N. O. C.; Graf, N. K.; Leutwyler, S.; Hättig, C. Benchmarks for 0-0 Transitions of Aromatic Organic Molecules: DFT/B3LYP, ADC(2), CC2, SOS-CC2 and SCS-CC2 Compared to High-resolution GasPhase Data. Phys. Chem. Chem. Phys. 2013, 15, 66236630.

(133) Smith, C. E.; King, R. A.; Crawford, T. D. Coupled Cluster Methods Including Triple Excitations for Excited States of Radicals. J. Chem. Phys. 2005, 122, 054110 .

(134) Mach, T. J.; King, R. A.; Crawford, T. D. A Coupled Cluster Benchmark Study of the Electronic Spectrum of the Allyl Radical. J. Phys. Chem. A 2010, 114, 8852-8857. 
(135) Aquilante, F.; Jensen, K. P.; Roos, B. O. The Allyl Radical Revisited: a Theoretical Study of the Electronic Spectrum. Chemical Physics Letters 2003, 380, 689-698.

(136) Gasser, M.; Frey, J. A.; Hostettler, J. M.; Bach, A.; Chen, P. Vibronic Structure of the $3 s$ and $3 p$ Rydberg States of the Allyl Radical. J. Phys. Chem. A 2010, 114, 4704-4711.

(137) Castiglioni, L.; Bach, A.; Chen, P. Spectroscopy and Dynamics of $A\left[{ }^{2} B_{1}\right]$ Allyl Radical. Phys. Chem. Chem. Phys. 2006, 8, 2591-2598.

(138) Gasser, M.; Schulenburg, A. M.; Dietiker, P. M.; Bach, A.; Merkt, F.; Chen, P. Single-Photon and Resonance-Enhanced Multiphoton Threshold Ionization of the Allyl Radical. J. Chem. Phys. 2009, 131, 014304.

(139) Maurice, D.; Head-Gordon, M. Configuration Interaction with Single Substitutions for Excited States of Open-Shell Molecules. Int. J. Quantum Chem. 1995, 56, 361-370.

(140) Ornellas, F.; Machado, F.; Roberto-Neto, O. A Theoretical Study of the Molecules $\mathrm{BeF}$ and $\mathrm{BeF}^{+}$in their Lowest-Lying Electronic States. Mol. Phys. 1992, 77, 1169-1185.

(141) El-Kork, N.; el kher, N. A.; Korjieh, F.; Chtay, J. A.; Korek, M. Electronic Structure of the Polar Molecules XF (X: Be, Mg, Ca) with Rovibrational and Dipole Moment Calculations. SpectroChim. Acta A 2017, 177, 170-196.

(142) Pitarch-Ruiz, J.; Sánchez-Marín, J.; Velasco, A. M. Full Configuration Interaction Calculation of the Low Lying Valence and Rydberg States of BeH. J. Comput. Chem. 2008, 29, 523-532.

(143) Perić, M.; Ostojić, B.; Peyerimhoff, S. D. Ab initio Calculation of the Potential Surfaces and the Electronic Transition Moments for the Valence and Rydberg Doublet Electronic States of $\mathrm{BH}_{2}$. Z. Physik D. 1995, 34, 241-249.

(144) Sunahori, F. X.; Gharaibeh, M.; Clouthier, D. J.; Tarroni, R. BH2 Revisited: New, Extensive Measurements of Laser-Induced Fluorescence Transitions and $\mathrm{Ab}$ Initio Calculations of Near-Spectroscopic Accuracy. J. Chem. Phys. 2015, 142, 174302.

(145) Szalay, P. G.; Gauss, J. Spin-Restricted Open-Shell Coupled-Cluster Theory for Excited States. J. Chem. Phys. 2000, 112, 4027-4036.

(146) Kállay, M.; Gauss, J.; Szalay, P. G. Analytic First Derivatives for General Coupled-Cluster and Configuration Interaction Models. J. Chem. Phys. 2003, 119, 2991-3004.

(147) Kállay, M.; Gauss, J. Calculation of ExcitedState Properties Using General Coupled-Cluster and Configuration-Interaction Models. J. Chem. Phys. 2004, 121, 9257-9269.

(148) Woodcock, H. L.; Wesolowski, S. S.; Yamaguchi, Y.; Schaefer, H. F. A Systematic Study of the $\tilde{X}^{2} B_{1}, \tilde{A}^{2} A_{1}$, and $\tilde{B}^{2} B_{2}$ States of the Neutral Radical $\mathrm{PH}_{2}$. J. Phys. Chem. A 2001, 105, 5037-5045.
(149) Yurchenko, S.; Thiel, W.; Jensen, P.; Bunker, P. Rotation-Vibration Energy Level Clustering in the $\tilde{X}^{2} B_{1}$ Ground Electronic State of $\mathrm{PH}_{2}$. J. Mol. Spectrosc. 2006, 239, 160-173.

(150) Jakubek, Z. J.; Bunker, P. R.; Zachwieja, M.; Nakhate, S. G.; Simard, B.; Yurchenko, S. N.; Thiel, W.; Jensen, P. A Dispersed Fluorescence and ab initio Investigation of the $\tilde{X}^{2} B_{1}$ and $\tilde{A}^{2} A_{1}$ Electronic States of the $\mathrm{PH}_{2}$ Molecule. J. Chem. Phys. 2006, 124, 094306.

(151) Slipchenko, L. V.; Krylov, A. I. Spin-Conserving and Spin-Flipping Equation-of-Motion Coupled-Cluster Method with Triple Excitations. J. Chem. Phys. 2005, 123, 084107.

(152) Hirata, S. Higher-Order Equation-of-Motion Coupled-Cluster Methods. J. Chem. Phys. 2004, 121, 51-59.

(153) Fan, P.-D.; Kamiya, M.; Hirata, S. ActiveSpace Equation-of-Motion Coupled-Cluster Methods through Quadruples for Excited, Ionized, and Electron-Attached States. J. Chem. Theory Comput. 2007, 3, 1036-1046.

(154) Mebel, A. M.; Lin, S.-H. Excited Electronic States of the Methyl Radical. Ab Initio Molecular Orbital Study of Geometries, Excitation Energies and Vibronic Spectra. Chem. Phys. 1997, 215, 329-341.

(155) Zanchet, A.; Bañares, L.; Senent, M. L.; GarcíaVela, A. An ab initio Study of the Ground and Excited Electronic Statesof the Methyl Radical. Phys. Chem. Chem. Phys. 2016, 18, 33195-33203.

(156) Settersten, T. B.; Farrow, R. L.; Gray, J. A. Coherent Infrared-Ultraviolet Double-Resonance Spectroscopy of $\mathrm{CH}_{3}$. Chem. Phys. Lett. 2003, 370, 204-210.

(157) Hudgens, J. W.; DiGiuseppe, T. G.; Lin, M. C. Two Photon Resonance Enhanced Multiphoton Ionization Spectroscopy and State Assignments of the Methyl Radical. J. Chem. Phys. 1983, 79, 571-582.

(158) Fu, H. B.; Hu, Y. J.; Bernstein, E. R. IR/UV Double Resonant Spectroscopy of the Methyl Radical: Determination of $v_{3}$ in the $3 p_{z}$ Rydberg State. J. Chem. Phys. 2005, 123, 234307.

(159) Yazidi, O.; Gritli, H.; Chambaud, G. Electronic Structure and Reactivity of the $\mathrm{CNO} / \mathrm{NCO} / \mathrm{CON}$ Isomers. Mol. Phys. 2005, 103, 3321-3336.

(160) heng Shi, D.; tao Li, W.; feng Sun, J.; lue Zhu, Z. MRCI Study on Spectroscopic and Molecular Properties of Several Low-Lying Electronic States of the CN Radical. J. Quant. Spectrosc. Radiat. Transf. 2011, 112, 2335-2346.

(161) Bao, J. J.; Gagliardi, L.; Truhlar, D. G. Multiconfiguration Pair-Density Functional Theory for Doublet Excitation Energies and Excited State Geometries: the Excited States of CN. Phys. Chem. Chem. Phys. 2017, 19, 30089-30096.

(162) Léonard, C.; Chambaud, G. Ab initio Study of the First Excited State $A^{2} \Sigma^{+}$and of the Transition $A^{2} \Sigma^{+} \leftarrow$ $X^{2} \Pi$ of CNO. Chem. Phys. Lett. 2008, 458, 24-28.

(163) Wu, M.; Northrup, F. J.; Sears, T. J. Study of RennerTeller, Spin-Orbit, and FermiâĂ ̌̌Resonance Inter- 
actions in $\tilde{X}^{2} \Pi\left(v_{1} v_{2} 0\right)$ Levels of NCO by Stimulated Emission Pumping Spectroscopy. J. Chem. Phys. 1992, 97, 4583-4595.

(164) Dixon, R. N. A ${ }^{2} \Pi-^{2} \Pi$ Electronic Band System of the Free NCO Radical. Canadian Journal of Physics 1960, 38, 10-16.

(165) Baraille, I.; Larrieu, C.; Dargelos, A.; Chaillet, M. A Variational Approach of the Calculation of FranckCondon Factors: the $\mathrm{F}_{2} \mathrm{BO}$ Emission Spectrum. Chem. Phys. 2002, 282, 9-20.

(166) Li, X. J.; Wang, M. S.; Yang, C. L.; He, D.; Ma, X. G. The Theoretical Study of the Ground and Excited States Properties for $\mathrm{F}_{2} \mathrm{BO}$ and $\mathrm{H}_{2} \mathrm{BO}$ Free Radicals. AIP Advances 2018, 8, 055021.

(167) Grimminger, R.; Sheridan, P. M.; Clouthier, D. J. An Experimental and $\mathrm{Ab}$ Initio Study of the Electronic Spectrum of the Jet-Cooled $\mathrm{F}_{2} \mathrm{BO}$ Free Radical. $J$. Chem. Phys. 2014, 140, 164302.

(168) Jin, B.; Sheridan, P. M.; Clouthier, D. J. Applied Quantum Chemistry: Spectroscopic Detection and Characterization of the $\mathrm{F}_{2} \mathrm{BS}$ and $\mathrm{Cl}_{2} \mathrm{BS}$ Free Radicals in the Gas Phase. J. Chem. Phys. 2015, 142, 124301.

(169) Clouthier, D. J. In Search of the $\mathrm{X}_{2} \mathrm{BO}$ and $\mathrm{X}_{2} \mathrm{BS}$ ( $\mathrm{X}=\mathrm{H}, \mathrm{F}$ ) Free Radicals: Ab Initio Studies of their Spectroscopic Signatures. J. Chem. Phys. 2014, 141, 244309.

(170) Kus, T.; Bartlett, R. J. Different Equation-of-Motion Coupled Cluster Methods with Different Reference Functions: The formyl Radical. J. Chem. Phys. 2008, 129, 104301.

(171) Serrano-Andrés, L.; Forsberg, N.; Malmqvist, P.-Å. Vibronic Structure in Triatomic Molecules: The Hydrocarbon Flame Bands of the Formyl Radical (HCO). A Theoretical Study. J. Chem. Phys. 1998, 108, 72027216.

(172) Ndengué, S. A.; Dawes, R.; Guo, H. A new set of Potential Energy Surfaces for HCO: Influence of RennerTeller Coupling on the Bound and Resonance Vibrational States. J. Chem. Phys. 2016, 144, 244301.

(173) Morgan, W. J.; Fortenberry, R. C. Quartic Force Fields for Excited Electronic States: Rovibronic Reference Data for the $1^{2} A^{\prime}$ and $1^{2} A$ " States of the Isoformyl Radical, HOC. SpectroChim. Acta A 2015, 135, 965972.

(174) Gharaibeh, M. A.; Clouthier, D. J.; Tarroni, R. Heavy Atom Nitroxyl Radicals. VI. The Electronic Spectrum of Jet-Cooled $\mathrm{H}_{2} \mathrm{PO}$, the Prototypical Phosphoryl Free Radical. J. Chem. Phys. 2011, 135, 214307.

(175) Grimminger, R. A.; Clouthier, D. J.; Tarroni, R. Heavy Atom Nitroxyl Radicals. V. An Experimental and $a b$ initio Study of the Previously Unknown $\mathrm{H}_{2} \mathrm{PS}$ Free Radical. J. Chem. Phys. 2011, 135, 214306.

(176) Cai, Z.-L. Ab Initio Study of the Low-Lying Electronic States of the $\mathrm{CH}_{2} \mathrm{NO}_{2}$ Radical. Int. J. Quantum Chem. 1994, 49, 781-788.

(177) Metz, R. B.; Cyr, D. R.; Neumark, D. M. Study of the ${ }^{2} B_{1}$ and ${ }^{2} A_{2}$ States of Nitromethyl Free Radical via Ultraviolet Photoelectron Spectroscopy of the $\mathrm{CH}_{2} \mathrm{NO}_{2}^{-}$ Anion. J. Phys. Chem. 1991, 95, 2900-2907.
(178) Cyr, D. R.; Leahy, D. J.; Osborn, D. L.; Continetti, R. E.; Neumark, D. M. Fast Beam Photodissociation of the $\mathrm{CH}_{2} \mathrm{NO}_{2}$ Radical. J. Chem. Phys. 1993, 99, 8751-8764.

(179) Forster, R.; Hippler, H.; Hoyermann, K.; Rohde, G.; Harding, L. B. REMPI Mass Spectrum of the $\mathrm{OH}$ Radical in the Gas Phase. Chem. Phys. Lett. 1991, $183,465-470$.

(180) Wang, J.-H.; Chang, H.-C.; Chen, Y.-T. Theoretical Study of Isomeric Structures and Low-Lying Electronic States of the Vinyl Radical $\mathrm{C}_{2} \mathrm{H}_{3}$. Chem. Phys. 1996, 206, 43-56.

(181) Mebel, A. M.; Chen, Y.-T.; Lin, S.-H. Ab initio Molecular Orbital Study of Excited Electronic States of the Vinyl Radical. Chem. Phys. Lett. 1997, 275, 19-27.

(182) Koziol, L.; Levchenko, S. V.; Krylov, A. I. Beyond Vinyl: Electronic Structure of Unsaturated Propen-1yl, Propen-2-yl, 1-Buten-2-yl, and trans-2-Buten-2-yl Hydrocarbon Radicals. J. Phys. Chem. A 2006, 110, 2746-2758.

(183) Pibel, C. D.; McIlroy, A.; Taatjes, C. A.; Alfred, S.; Patrick, K.; Halpern, J. B. The Vinyl Radical $\left(\tilde{A}^{2} A " \leftarrow\right.$ $\left.\tilde{X}^{2} A^{\prime}\right)$ Spectrum Between 530 and $415 \mathrm{~nm}$ Measured by Cavity Ring-Down Spectroscopy. J. Chem. Phys. 1999, 110, 1841-1843. 


\section{Graphical TOC Entry}

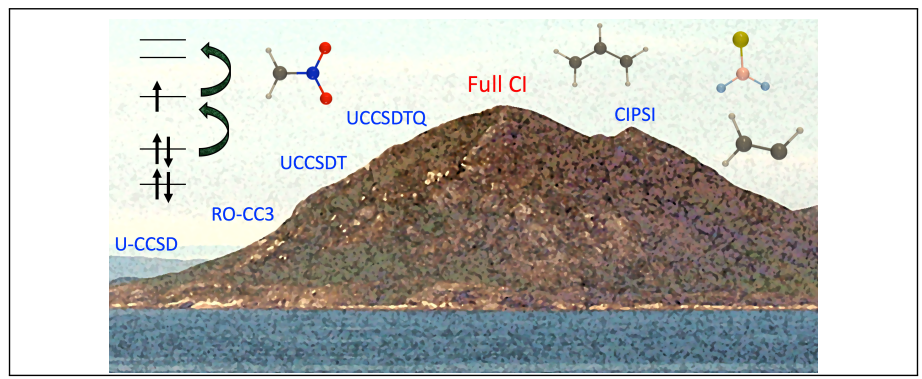

